\title{
Complex Dynamical Behaviors in a Predator-Prey System with Generalized Group Defense and Impulsive Control Strategy
}

\author{
Shunyi Li \\ Department of Mathematics, Qiannan Normal College for Nationalities, Duyun, Guizhou 558000, China \\ Correspondence should be addressed to Shunyi Li; lishunyi19820425@163.com
}

Received 1 November 2012; Accepted 20 May 2013

Academic Editor: Patricia J. Y. Wong

Copyright (C) 2013 Shunyi Li. This is an open access article distributed under the Creative Commons Attribution License, which permits unrestricted use, distribution, and reproduction in any medium, provided the original work is properly cited.

\begin{abstract}
A predator-prey system with generalized group defense and impulsive control strategy is investigated. By using Floquet theorem and small amplitude perturbation skills, a local asymptotically stable prey-eradication periodic solution is obtained when the impulsive period is less than some critical value. Otherwise, the system is permanent if the impulsive period is larger than the critical value. By using bifurcation theory, we show the existence and stability of positive periodic solution when the pest eradication lost its stability. Numerical examples show that the system considered has more complicated dynamics, including (1) high-order quasiperiodic and periodic oscillation, (2) period-doubling and halving bifurcation, (3) nonunique dynamics (meaning that several attractors coexist), and (4) chaos and attractor crisis. Further, the importance of the impulsive period, the released amount of mature predators and the degree of group defense effect are discussed. Finally, the biological implications of the results and the impulsive control strategy are discussed.
\end{abstract}

\section{Introduction}

In population dynamics, a functional response of the predator to the prey density refers to the change in the density of prey attached per unit time per predator as the prey density changes and it is assumed to be monotonically increasing in most predator-prey systems. For example, Holling type I, II, and III functional response [1]

$$
\begin{gathered}
f_{1}(x, y)=r x, \quad f_{2}(x, y)=\frac{r x}{a+b x}, \\
f_{3}(x, y)=\frac{r x^{2}}{a+b x^{2}},
\end{gathered}
$$

and the sigmoidal type response function [2]

$$
f_{4}(x, y)=\frac{r x^{2}}{(a+x)(b+x)},
$$

and Ivlev type response function [3]

$$
f_{5}(x, y)=r\left(1-e^{-a x}\right) .
$$

The previous functional responses are prey dependent. But, both predator and prey densities have an effect on the response, such as Beddington-DeAngelis functional response $[4,5]$

$$
f_{6}(x, y)=\frac{r x}{a+b x+c y}
$$

and modified Holling type II and type III response functions [6]

$$
\begin{aligned}
& f_{7}(x, y)=\frac{r x}{(a+b x)(b+y)}, \\
& f_{8}(x, y)=\frac{r x^{2}}{\left(a+b x^{2}\right)(b+y)} .
\end{aligned}
$$

However, some experimental and observational evidence shown that the functional response is not always monotonically increasing, such as Holling type IV [7]

$$
f_{9}(x, y)=\frac{r x}{a+b x+c x^{2}}
$$

and $f_{10}(x, y)=\alpha x e^{-\beta x}[8]$. Group defense is a term used to describe the phenomenon whereby predation is decreased, or even prevented altogether, due to the increased ability of 
the prey to better defend or disguise itself when it exists in enough large numbers [9-11]. The buffalo group defense was modeled using a generalized group defense in [12],

$$
f_{11}(x, y)=\frac{\alpha x}{1+h x^{\beta}}
$$

where $\beta$ is a positive integer whose value determines the degree of antipredator behavior and group defense.

Recently, it is of great interest to investigate complex dynamics for impulsive perturbations in populations dynamics. In particular, the impulsive prey-predator population models have been investigated by many researchers. The results of studies of the dynamics of a predator-prey model with nonmonotonic functional response, such as Holling type IV functional response with respect to an impulsive control strategy, were presented in [13-24]. To the best of our knowledge, there are few papers studying the group defense predator-prey with impulsive effect, where the antipredator behavior and group defense effect described by nonmonotonic functional response. Zhang et al. [25] considered a predator-prey system with defensive ability of prey by Holling type IV functional response and impulsive perturbations on the predator:

$$
\begin{gathered}
x^{\prime}(t)=r x(t)\left(1-\frac{x(t)}{k}\right)-\frac{x(t) y(t)}{a_{1}+x^{2}(t)}, \quad t \neq n T, \\
y^{\prime}(t)=y(t)\left(-d+\frac{\mu x(t)}{a_{1}+x^{2}(t)}\right), \\
x\left(n T^{+}\right)=x(n T), \quad y\left(n T^{+}\right)=y(n T)+\tau, \quad t=n T . \\
X\left(0^{+}\right)=x_{0}=\left(x^{0}, y^{0}\right)^{T},
\end{gathered}
$$

The conditions for the local asymptotically stable preyeradication periodic solution and permanence of the system are obtained; a series of complex phenomena are displayed by numerical simulation. Furthermore, based on this work, Pei et al. [26] investigated a one-prey multi-predator model with defensive ability of the prey by introducing impulsive biological control strategy:

$$
\begin{gathered}
x^{\prime}(t)=r x(t)\left(1-\frac{x(t)}{k}\right)-\sum_{i=1}^{m} \frac{x(t) y(t)}{a_{i}+x^{2}(t)}, \\
y_{i}^{\prime}(t)=y_{i}(t)\left(\sum_{i=1}^{m} \frac{\mu_{i} x(t)}{a_{i}+x^{2}(t)}-d_{i}\right), \\
x\left(n T^{+}\right)=x(n T), \quad y_{i}\left(n T^{+}\right)=y_{i}(n T)+p_{i}, \\
X_{0}=\left(x\left(0^{+}\right), y_{1}\left(0^{+}\right), \ldots, y_{m}\left(0^{+}\right)\right)^{T}=\left(x_{0}, y_{01}, \ldots, y_{0 m}\right)^{T}, \\
t=n T .
\end{gathered}
$$

And it shown that the multi-predator impulsive control strategy is more effective than the classical one and makes the dynamical behaviors of the system more complex. Recently, a predator-prey system with impulsive effect and group defense with the nonmonotone function $f_{10}(x, y)=\alpha x e^{-\beta x}$ was studied by Li et al. [27],

$$
\begin{gathered}
x^{\prime}(t)=x(t)(a-b x(t))-\alpha x(t) y(t) e^{-\beta x(t)}, \quad t \neq n T, \\
y^{\prime}(t)=k \alpha x(t) y(t) e^{-\beta x(t)}-y(t) d, \\
\Delta x(t)=-p x(t), \quad t=n T . \\
\Delta y(t)=q,
\end{gathered}
$$

They proved that there exists a locally stable pest-eradication periodic solution when the impulsive period is less than certain critical values; otherwise, the system is permanent. Some complicated dynamics, such as quasiperiodic oscillation, bifurcation, and attractor crisis, were shown by numerical simulations.

In this paper, we study a predator-prey system with impulsive effect and generalized group defense with the nonmonotone function $f_{11}(x, y)=\alpha x /\left(1+h x^{\beta}\right)$ :

$$
\begin{gathered}
x^{\prime}(t)=x(t)(a-b x(t))-\frac{\alpha x(t)}{1+h x^{\beta}(t)} y(t), \\
y^{\prime}(t)=k \frac{\alpha x(t)}{1+h x^{\beta}(t)} y(t)-d y(t), \\
\Delta x(t)=-p_{1} x(t), \quad t \neq n T, \quad t=n T, \\
\Delta y(t)=-p_{2} y(t)+q, \quad
\end{gathered}
$$

where $x(t)$ and $y(t)$ represent the prey and the predator populations at time $t$, respectively; $a, b, \alpha, h, \beta$, and $k$ are positive. $a$ is the intrinsic rate of increase of the prey and $d$ is the death rate of the predator, $a / b$ is the carrying capacity of the prey, $\beta>1$ is the degree of anti-predator behavior and group defense, and $k(0<k<1)$ is the rate of conversing prey into predator. $\Delta x(t)=x\left(t^{+}\right)-x(t), \Delta y(t)=y\left(t^{+}\right)-y(t), T$ is the periodic of the impulse for predator in order to eradicate target pests, protect nontarget pest (or harmless insect) from extinction and drive target pest to extinction, or control target pest at acceptably low level to prevent an increasing pest population from causing an economic loss. $n \in \mathbb{N}_{+}, \mathbb{N}_{+}=$ $\{1,2, \ldots\}, p_{i}>0(i=1,2)$ is the proportionality constant which represents the rate of mortality due to the applied pesticide; for example, impulsive reduction of the population is possible by harvesting or by poisoning with chemicals used in agriculture. $q>0$ is the number of predators released each time, for example, by artificial breeding of the species or release of some species.

The paper is arranged as follows. In Section 2, some notations and Lemmas are given. In Section 3, using the Floquet theory of impulsive equation and small amplitude perturbation skills, we will prove the local stability of preyeradication periodic solution when the impulsive period is less than some critical value and give the condition of permanence. In Section 4, by using bifurcation theory, the existence and stability of positive periodic solution are studied when $T$ is close to the critical value $T_{0}$. In Section 5 , the results of numerical examples are shown, and some rich dynamic behaviors are obtained; the effects of the impulsive period, the released amount of mature predators and 
the coefficient of group defense effect are discussed. Finally, the conclusions are discussed briefly in Section 6 .

\section{Preliminaries}

In this section, we will give some definitions, notations, and lemmas which will be useful for our main results.

Let $\mathbb{R}_{+}=[0, \infty), \mathbb{R}_{+}^{2}=\left\{x \in \mathbb{R}^{2} \mid x \geq 0\right\}$. Denote by $f=\left(f_{1}, f_{2}\right)$ the map defined by the right hand of the first two equations of system (11), and denote by $\mathbb{N}$ the set of all nonnegative integers. Let $V: \mathbb{R}_{+} \times \mathbb{R}_{+}^{2} \rightarrow \mathbb{R}_{+}$, then $V$ is said to belong to class $V_{0}$ if

(1) $V$ is continuous in $(t, x) \in(n T,(n+1) T] \times \mathbb{R}_{+}^{2}$ and for each $x \in \mathbb{R}_{+}^{2}, n \in \mathbb{N}, \lim _{(t, y) \rightarrow\left(n T^{+}, x\right)} V(t, y)=$ $V\left(n T^{+}, x\right)$ exists,

(2) $V$ is locally Lipschitzian in $x$.

Definition 1. Let $V \in V_{0}$; then for $(t, x) \in(n T,(n+1) T] \times$ $\mathbb{R}_{+}^{2}$, the upper right derivative of $V(t, x)$ with respect to the impulsive differential system (11) is defined as

$$
D^{+} V(t, x)=\lim _{h \rightarrow 0^{+}} \sup \frac{1}{h}[V(t+h, x+h f(t, x))-V(t, x)] .
$$

Definition 2. System (11) is said to be permanent if there exist two positive constants $m, M$ and $T_{0}$ such that each positive solution $(x(t), y(t))$ of the system (11) satisfies $m \leq x(t) \leq$ $M, m \leq y(t) \leq M$, for all $t>T_{0}$.

The solution of system (11) is a piecewise continuous function $x: \mathbb{R}_{+} \mapsto \mathbb{R}_{+}^{2}, x(t)$ is continuous on $(n T,(n+$ 1)T], $n \in \mathbb{N}$, and $x\left(n T^{+}\right)=\lim _{t \rightarrow n T^{+}} x(t)$ exists; the smoothness properties of $f$ guarantee the global existence and uniqueness of solutions of system (11); for details see $[28,29]$. The following lemma is obvious.

Lemma 3. Let $X(t)$ be a solution of system (11) with $X\left(0^{+}\right) \geq 0$; then $X(t) \geq 0$ for all $t \geq 0$ and further $X(t)>0$ for all $t \geq 0$ if $X\left(0^{+}\right)>0$.

And we will use the following important comparison theorem on impulsive differential equation [29].

Lemma 4. Suppose $V \in V_{0}$. Assume that

$$
\begin{array}{ll}
D^{+} V(t, x) \leq g(t, V(t, x)), & t \neq n T, \\
V\left(t, x\left(t^{+}\right)\right) \leq \psi_{n}(V(t, x)), & t=n T,
\end{array}
$$

where $g: \mathbb{R}_{+} \times \mathbb{R}_{+} \mapsto \mathbb{R}$ is continuous in $(n T,(n+1) T] \times \mathbb{R}_{+}$, and for $u \in \mathbb{R}_{+}, n \in \mathbb{N}, \lim _{(t, y) \rightarrow\left(n T^{+}, u\right)}=g\left(n T^{+}, u\right)$ exists; $\psi_{n}: \mathbb{R}_{+} \rightarrow \mathbb{R}_{+}$is nondecreasing. Let $r(t)$ be the maximal solution of the scalar impulsive differential equation

$$
\begin{gathered}
u^{\prime}(t)=g(t, u(t)), \quad t \neq n T, \\
u\left(t^{+}\right)=\psi_{n}(u(t)), \quad t=n T, \\
u\left(0^{+}\right)=u_{0},
\end{gathered}
$$

existing on $[0, \infty)$. Then $V\left(0^{+}, x_{0}\right) \leq u_{0}$ implies that $V(t, x(t)) \leq r(t), t \geq 0$ where $X(t)$ is any solution of system (11).

Finally, we give some basic properties about the following subsystem of system (11):

$$
\begin{gathered}
y^{\prime}(t)=-d y(t), \quad t \neq n T, \\
\Delta y(t)=-p_{2} y(t)+q, \quad t=n T, \\
y\left(0^{+}\right)=y_{0} \geq 0 .
\end{gathered}
$$

Clearly, when $t \in(n T,(n+1) T], n \in \mathbb{N}$,

$$
\begin{aligned}
& y^{*}(t)=\frac{q \exp [-d(t-n T)]}{1-\left(1-p_{2}\right) \exp (-d T)}, \\
& y^{*}(0)=\frac{q}{1-\left(1-p_{2}\right) \exp (-d T)},
\end{aligned}
$$

is a positive periodic solution of system (15). Since

$$
\begin{aligned}
y(t)= & \left(y\left(0^{+}\right)-\frac{q}{1-\left(1-p_{2}\right) \exp (-d T)}\right) \\
& \times \exp (-d t)+y^{*}(t)
\end{aligned}
$$

is the solution of system (15) with initial value $y_{0} \geq 0$, where $t \in(n T,(n+1) T], n \in \mathbb{N}$; then one can get the following.

Lemma 5. Let $y^{*}(t)$ be a positive periodic solution of system; (15) and every solution $y(t)$ of system (15) with $y_{0} \geq 0$, one has $\left|y(t)-y^{*}(t)\right| \rightarrow 0$, when $t \rightarrow \infty$. tion

Therefore, one obtains the pest-eradication periodic solu-

$$
\left(0, y^{*}(t)\right)=\left(0, \frac{q \exp [-d(t-n T)]}{1-\left(1-p_{2}\right) \exp (-d T)}\right)
$$

for $t \in(n T,(n+1) T]$.

\section{Extinction and Permanence}

Firstly, we study the stability of prey-eradication periodic solution.

Theorem 6. Let $X(t)=(x(t), y(t))$ be any solution of system (11); then $X(t)=\left(0, y^{*}(t)\right)$ is locally asymptotically stable provided that

$$
a T-\frac{\alpha q[1-\exp (-d T)]}{d\left[1-\left(1-p_{2}\right) \exp (-d T)\right]}<\ln \left(\frac{1}{1-p_{1}}\right) .
$$

Proof. The local stability of periodic solution $X(t)=$ $\left(0, y^{*}(t)\right)$ may be determined by considering the behavior of small amplitude perturbations of the solution. Consider

$$
x(t)=u(t), \quad y(t)=y^{*}(t)+v(t) .
$$

There may be written

$$
\left(\begin{array}{l}
u(t) \\
v(t)
\end{array}\right)=\Phi(t)\left(\begin{array}{l}
u(0) \\
v(0)
\end{array}\right), \quad 0 \leq t<T
$$


where $\Phi(t)$ satisfies

$$
\frac{\mathrm{d} \Phi}{\mathrm{d} t}=\left(\begin{array}{cc}
a-\alpha y^{*}(t) & 0 \\
k \alpha y^{*}(t) & -d
\end{array}\right) \Phi(t),
$$

and $\Phi(0)=I$, the identity matrix. The linearization of the third and fourth equations of system (11) becomes

$$
\left(\begin{array}{l}
u\left(n T^{+}\right) \\
v\left(n T^{+}\right)
\end{array}\right)=\left(\begin{array}{cc}
1-p_{1} & 0 \\
0 & 1-p_{2}
\end{array}\right)\left(\begin{array}{l}
u(n T) \\
v(n T)
\end{array}\right) .
$$

Hence, if both eigenvalues of

$$
M=\left(\begin{array}{cc}
1-p_{1} & 0 \\
0 & 1-p_{2}
\end{array}\right) \Phi(t)
$$

have absolute values less than one, then the periodic solution $X(t)=\left(0, y^{*}(t)\right)$ is locally stable. Since all eigenvalues of $M$ are

$$
\begin{aligned}
& \mu_{1}=\left(1-p_{2}\right) \exp (-d T)<1, \\
& \mu_{2}=\left(1-p_{1}\right) \exp \left(\int_{0}^{T}\left(a-\alpha y^{*}(t)\right) d t\right),
\end{aligned}
$$

$\mu_{2}<1$ if and only if

$$
a T-\frac{\alpha q[1-\exp (-d T)]}{d\left[1-\left(1-p_{2}\right) \exp (-d T)\right]}<\ln \left(\frac{1}{1-p_{1}}\right) .
$$

According to Floquet theory [28] of impulsive differential equation, the prey-eradication solution $X(t)=\left(0, y^{*}(t)\right)$ is locally stable. This completes the proof.

Theorem 7. There exists a constant $M>0$, such that $x(t) \leq$ $M, y(t) \leq M$ for each solution $X(t)=(x(t), y(t))$ of system (11) with all t being large enough.

Proof. Let $V(t)=k x(t)+y(t)$. It is clear that $V \in V_{0}$. We calculate the upper right derivative of $V(t, x)$ along a solution of system (11) and get the following impulsive differential equation:

$$
\begin{aligned}
& \left.D^{+} V(t)\right|_{(11)}+L V(t) \\
& \quad=k x(a+L-b x)-(d-L) y, \quad t \neq n T, \\
& V\left(t^{+}\right) \leq V(t)+q, \quad t=n T .
\end{aligned}
$$

Let $0<L<d$; then $k x(a+L-b x)-(d-L) y$ is bounded. Select $L_{0}$ and $M_{0}$ such that

$$
\begin{gathered}
D^{+} V(t) \leq-L_{0} V(t)+M_{0}, \quad t \neq n T, \\
V\left(t^{+}\right) \leq V(t)+q, \quad t=n T,
\end{gathered}
$$

where $L_{0}$ and $M_{0}$ are two positive constants. According to Lemma 4 , we have

$$
\begin{aligned}
V(t) \leq & \frac{M_{0}}{L_{0}}+\left(V\left(0^{+}\right)-\frac{M_{0}}{L_{0}}\right) \\
& \times \exp \left(-L_{0} t\right)-\frac{q}{1-\exp \left(-L_{0} T\right)} \exp \left(-L_{0} t\right) \\
& +\frac{q}{1-\exp \left(-L_{0} T\right)} \exp \left(-L_{0}(t-n T)\right),
\end{aligned}
$$

where $t \in(n T,(n+1) T]$. Hence

$$
\lim _{t \rightarrow \infty} V(t) \leq \frac{M_{0}}{L_{0}}+\frac{q}{1-\exp \left(-L_{0} T\right)} .
$$

Therefore, $V(t, x)$ is ultimately bounded. We obtain that each positive solution of system (11) is uniformly ultimately bounded. This completes the proof. (11).

In the following, we investigate the permanence of system

Theorem 8. System (11) is permanent if

$$
a T-\frac{\alpha q[1-\exp (-d T)]}{d\left[1-\left(1-p_{2}\right) \exp (-d T)\right]}>\ln \left(\frac{1}{1-p_{1}}\right) .
$$

Proof. Suppose $X(t)=(x(t), y(t))$ is a solution of system (11) with $X_{0}>0$. From Theorem 7 we may assume that $x(t) \leq$ $M, y(t) \leq M$, and $M>a / b, t \geq 0$. Let

$$
m_{2}=\frac{q \exp (-d T)}{1-\left(1-p_{2}\right) \exp (-d T)}-\varepsilon_{2}, \quad \varepsilon_{2}>0 .
$$

According to Lemmas 4 and 5 , we have $y(t)>m_{2}$ for all $t$ large enough. In the following, we want to find $m_{1}$ such that $x_{1}(t) \geq m_{1}$ for all $t$ large enough. We will do it in the following two steps for convenience.

Step 1. Since

$$
a T-\frac{\alpha q[1-\exp (-d T)]}{d\left[1-\left(1-p_{2}\right) \exp (-d T)\right]}>\ln \left(\frac{1}{1-p_{1}}\right),
$$

we can select $m_{3}>0, \varepsilon_{1}>0$ small enough such that $m_{3}<$ $a / b, \delta=k \alpha m_{3}<d$, and

$$
\begin{aligned}
\sigma= & \left(a-b m_{3}-\alpha \varepsilon_{1}\right) T \\
& -\frac{\alpha q[1-\exp ((-d+\delta) T)]}{(d-\delta)\left[1-\left(1-p_{2}\right) \exp ((-d+\delta) T)\right]} \\
& -\ln \left(\frac{1}{1-p_{1}}\right)>0 .
\end{aligned}
$$

We will prove there exists $t_{1} \in(0, \infty)$ such that $x\left(t_{1}\right) \geq$ $m_{3}$. Otherwise, according to the above assumption, we get $y^{\prime}(t) \leq y(t)(-d+\delta)$, and by Lemmas 4 and 5 , we have $y(t) \leq z(t)$ and $z(t) \leq z^{*}(t)$, where

$$
\begin{array}{r}
z^{*}(t)=\frac{q \exp [(-d+\delta)(t-n T)]}{1-\left(1-p_{2}\right) \exp ((-d+\delta) T)}, \\
t \in(n T,(n+1) T],
\end{array}
$$

and $z(t)$ is the solution of the following equation:

$$
\begin{gathered}
z^{\prime}(t)=z(t)(-d+\delta), \quad t \neq n T, \\
\Delta z(t)=-p_{2} z(t)+q, \quad t=n T, \\
z\left(0^{+}\right)=y_{0} \geq 0 .
\end{gathered}
$$


Therefore, there exists a $T_{1}>0$ such that

$$
\begin{gathered}
y(t) \leq z(t) \leq z^{*}(t)+\varepsilon_{1}, \\
x^{\prime}(t) \geq x(t)\left(a-b m_{3}-\left(z^{*}(t)+\varepsilon_{1}\right)\right) .
\end{gathered}
$$

Let $N_{1} \in \mathbb{N}$ and let $N_{1} T \geq T_{1}$. We can get

$$
\begin{gathered}
x^{\prime}(t) \geq x(t)\left(a-b m_{3}-\left(z^{*}(t)+\varepsilon_{1}\right)\right), \quad t \neq n T, \\
\Delta x(t)=-p_{1} x(t), \quad t=n T .
\end{gathered}
$$

Integrating (38) on $(n T,(n+1) T]\left(n \geq N_{1}\right)$, we have

$$
\begin{aligned}
& x((n+1) T) \\
& \geq x\left(n T^{+}\right) \exp \left(\int_{n T}^{(n+1) T}\left(a-b m_{3}-\alpha\left(z^{*}(t)+\varepsilon_{1}\right)\right) d t\right) \\
& =\left(1-p_{1}\right) x(n T) \\
& \quad \times \exp \left[\left(a-b m_{3}-\alpha \varepsilon_{1}\right) T\right. \\
& \left.\quad-\frac{\alpha q[1-\exp ((-d+\delta) T)]}{(d-\delta)\left[1-\left(1-p_{2}\right) \exp ((-d+\delta) T)\right]}\right]
\end{aligned}
$$$$
=x(n T) \exp (\sigma) \text {. }
$$

Then $x\left(\left(N_{1}+h\right) T\right) \geq x\left(N_{1} T\right) \exp (h \sigma) \rightarrow \infty$ as $h \rightarrow \infty$, which is a contradiction to the boundedness of $x(t)$. Hence there exists a $t_{1}>0$ such that $x\left(t_{1}\right) \geq m_{3}$.

Step 2. If $x\left(t_{1}\right) \geq m_{3}$ for all $t \geq t_{1}$, then our aim is obtained.

Hence we only need to consider those solutions which leave the region $R=\left\{X(t) \in \mathbb{R}_{2}^{+}: x(t)<m_{3}\right\}$ and reenter again. Let $t^{*}=\inf _{t \geq t_{1}}\left\{x(t)<m_{3}\right\}$. Then $t^{*}$ is impulsive point or nonimpulsive point.

Case 1. If $t^{*}$ is impulsive point, there exist a $n_{1} \in \mathbb{N}$ such that $t^{*}=n_{1} T$. Then $x(t) \geq m_{3}$ for $t \in\left[t_{1}, t^{*}\right]$ and

$$
\left(1-p_{1}\right) m_{3} \leq x\left(t^{*+}\right)=\left(1-p_{1}\right) x\left(t^{*}\right)<m_{3} .
$$

Choose $n_{2}, n_{3} \in \mathbb{N}$ such that

$$
\begin{gathered}
n_{2} T>T_{2}=\frac{\ln \left(\varepsilon_{1} /(M+q)\right)}{(-d+\delta)}, \\
(1-p)^{n_{2}+1} \exp \left(\left(n_{2}+1\right) \sigma_{1} T\right) \exp \left(n_{3} \sigma\right)>1,
\end{gathered}
$$

where $\sigma_{1}=a-b m_{3}-\alpha M<0$. Let $\bar{T}=\left(n_{2}+n_{3}\right) T$. Then, there exists a $t_{2} \in\left[\left(n_{1}+1\right) T,\left(n_{1}+1\right) T+\bar{T}\right]$ such that $x\left(t_{2}\right) \geq m_{3}$. Otherwise $x(t)<m_{3}, t \in\left[\left(n_{1}+1\right) T,\left(n_{1}+1\right) T+\bar{T}\right]$. Consider (36) with $z\left(\left(n_{1}+1\right) T^{+}\right)=y\left(\left(n_{1}+1\right) T^{+}\right)$; we have

$$
\begin{aligned}
z(t)= & \left(z\left(n_{1}+1\right) T^{+}-\frac{q}{1-\left(1-p_{2}\right) \exp ((-d+\delta) T)}\right) \\
& \times \exp \left[(-d+\delta)\left(t-\left(n_{1}+1\right) T\right)\right]+z^{*}(t),
\end{aligned}
$$

where $t \in(n T,(n+1) T], n_{1}+1 \leq n \leq\left(n_{1}+1\right)+n_{2}+n_{3}$. Then

$$
\begin{gathered}
\left|z(t)-z^{*}(t)\right|<(M+q) \exp \left(-(d-\delta) n_{2} T\right)<\varepsilon_{1}, \\
y(t) \leq z(t) \leq z^{*}(t)+\varepsilon_{1}
\end{gathered}
$$

for $\left(n_{1}+1+n_{2}\right) T \leq t \leq\left(n_{1}+1\right) T+\bar{T}$, which implies (39) holds for $\left(n_{1}+1+n_{2}\right) T \leq t \leq\left(n_{1}+1\right) T+\bar{T}$. As in step 1 , we have

$$
x\left(\left(n_{1}+1+n_{2}+n_{3}\right) T\right) \geq x\left(\left(n_{1}+1+n_{2}\right) T\right) \exp \left(n_{3} \sigma\right) .
$$

The first and third equations of system (11) given

$$
\begin{aligned}
& x^{\prime}(t)>x(t)\left(a-b m_{3}-\alpha M\right)=\sigma_{1} x(t), \quad t \neq n T, \\
& x\left(t^{+}\right)=\left(1-p_{1}\right) x(t), \quad t=n T .
\end{aligned}
$$

Integrating the above equation on $\left[t^{*},\left(n_{1}+1+n_{2}\right) T\right]$, we can get

$$
\begin{aligned}
& x\left(\left(n_{1}+1+n_{2}\right) T\right) \\
& \quad \geq(1-p)^{n_{2}+1} m_{3} \exp \left(\sigma_{1}\left(n_{2}+1\right) T\right),
\end{aligned}
$$

and thus

$$
\begin{aligned}
& x\left(\left(n_{1}+1+n_{2}+n_{3}\right) T\right) \\
& \quad \geq m_{3}(1-p)^{n_{2}+1} \exp \left(\left(n_{2}+1\right) \sigma_{1} T\right) \exp \left(n_{3} \sigma\right) \\
& \quad>m_{3},
\end{aligned}
$$

a contradiction.

Let $\bar{t}=\inf _{t \geq t^{*}}\left\{x(t) \geq m_{3}\right\}$; then $x(\bar{t}) \geq m_{3}$. For $t \in\left[t^{*}, \bar{t}\right)$, we have

$$
x(t) \geq m_{3} \exp \left(\sigma_{1}\left(1+n_{2}+n_{3}\right) T\right)(1-p)^{1+n_{2}+n_{3}} \triangleq m_{1} .
$$

For $t>\bar{t}$, the same arguments can be continued since $x(\bar{t}) \geq$ $m_{3}$.

Case 2. If $t^{*}$ is nonimpulsive point, then $x(t) \geq m_{3}$ for $t \in$ $\left[t_{1}, t^{*}\right)$ and $x\left(t^{*}\right)=m_{3}$; suppose $t^{*} \in\left(n_{1}^{\prime} T,\left(n_{1}^{\prime}+1\right) T\right), n_{1}^{\prime} \in \mathbb{N}$. There are two possible cases for $t \in\left(t^{*},\left(n_{1}^{\prime}+1\right) T\right)$.

Case 2.1. $x(t) \leq m_{3}$ for all $t \in\left(t^{*},\left(n_{1}^{\prime}+1\right) T\right)$. As in Step 1, we can prove that there must be a $t_{2}^{\prime} \in\left[\left(n_{1}^{\prime}+1\right) T,\left(n_{1}^{\prime}+1\right) T+\bar{T}\right]$ such that $x\left(t_{2}^{\prime}\right)>m_{3}$; Let $\bar{t}=\inf _{t \geq t^{*}}\left\{x(t) \geq m_{3}\right\}$, then $x(\bar{t}) \leq$ $m_{3}$ and $x(\bar{t})=m_{3}$. For $t \in\left[t^{*}, \bar{t}\right)$, we have

$$
\begin{aligned}
x(t) & \geq m_{3} \exp \left(\sigma_{1}\left(1+n_{2}+n_{3}\right) T\right)(1-p)^{1+n_{2}+n_{3}} \\
& \triangleq m_{1} .
\end{aligned}
$$

For $t>\bar{t}$, the same arguments can be continued since $x(\bar{t}) \geq$ $m_{3}$.

Case 2.2. There exists a $t \in\left(t^{*},\left(n_{1}^{\prime}+1\right) \bar{T}\right)$ such that $x(t)>m_{3}$. Let $\check{t}=\inf _{t>t^{*}}\left\{x(t)>m_{3}\right\}$; then $x(t) \leq m_{3}$ for $t \in\left(t^{*}, \check{t}\right)$ and 
$x(\check{t})=m_{3}$. For $t \in\left(t^{*}, \check{t}\right),(45)$ holds true; integrating (45) on $t \in\left(t^{*}, \check{t}\right)$, we have

$$
\begin{aligned}
x(t) & \geq x\left(t^{*}\right) \exp \left[\sigma_{1}\left(t-t^{*}\right)\right] \geq m_{3} \exp \left(\sigma_{1} T\right) \\
& >m_{1} .
\end{aligned}
$$

Since $x(\check{t}) \geq m_{3}$ for $t>\check{t}$, the same arguments can be continued.

Hence $x(t) \geq m_{1}$ for all $t>t_{1}$. The proof is completed.

Remark 9. Let

$$
\begin{aligned}
f(T)= & a T-\frac{\alpha q[1-\exp (-d T)]}{d\left[1-\left(1-p_{2}\right) \exp (-d T)\right]} \\
& -\ln \left(\frac{1}{1-p_{1}}\right) .
\end{aligned}
$$

Since $f(0)=-\ln \left(1 /\left(1-p_{1}\right)\right)<0, f(T) \rightarrow+\infty$ as $T \rightarrow+\infty$, and

$$
\begin{aligned}
f^{\prime \prime}(T)= & \left(\alpha q p _ { 2 } d \left[\left[1-\left(1-p_{2}\right) \exp (-d T)\right] \exp (-d T)\right.\right. \\
& \left.\left.+2\left(1-p_{2}\right) \exp (-2 d T)\right]\right) \\
& \times\left(\left[1-\left(1-p_{2}\right) \exp (-d T)\right]^{3}\right)^{-1} \\
> & 0,
\end{aligned}
$$

so $f(T)=0$ has a unique positive root, denoted by $T_{\max }$. From Theorems 6 and 8 we know that $T_{\max }$ is a threshold. If $T<T_{\max }$, then pest-eradication periodic solution $\left(0, y^{*}(t)\right)$ is asymptotically stable; if $T>T_{\max }$, then system (11) is permanent.

Remark 10. If $p_{1}=p_{2}=0, q=0$; that is, there are without taking any pest-management strategy, large numbers of preys (pest) would coexisting with predators (natural enemy). If $q=0,0<p_{1}, p_{2}<1$, that is, there is periodic spraying pesticide (or harvesting) only. Thus, we can easily obtain that $T_{\max }^{\prime}=\ln \left(1 /\left(1-p_{1}\right)\right) / a<T_{\max }$ is the threshold. If $p_{1}=$ $p_{2}=0, q>0$; that is, there is periodic releasing of predator (natural enemy) only, without periodic spraying pesticide (or harvesting). We can easily get that $T_{\max }^{\prime \prime}=\alpha q /(a d)<T_{\max }$ is the threshold. Comparing with the classic methods (such as biological control or chemical control), the integrated pest management (IPM) is a better one, since $T_{\max }>T_{\max }^{\prime}$ and $T_{\max }>T_{\max }^{\prime \prime}$. Some numerical examples will be given in Section 5.

\section{Bifurcation and Existence of Positive Periodic Solution}

In this section, we deal with the existence of a nontrivial periodic solution to system (11) near the prey-eradication periodic solution $\left(0, y^{*}(t)\right)$ via bifurcation.
Let $x_{1}(t)=y(t), x_{2}(t)=x(t)$; system (11) becomes as follows:

$$
\begin{aligned}
x_{1}^{\prime}(t) & =x_{1}(t)\left(\frac{k \alpha x_{2}(t)}{1+h x_{2}^{\beta}(t)}-d\right) \\
& \triangleq F_{1}\left(x_{1}(t), x_{2}(t)\right), \\
x_{2}^{\prime}(t) & =x_{2}(t)\left(a-b x_{2}(t)-\frac{\alpha x_{1}(t)}{1+h x_{2}^{\beta}(t)}\right) \\
& \triangleq F_{2}\left(x_{1}(t), x_{2}(t)\right),
\end{aligned}
$$

$t \neq n T$,

$$
\begin{aligned}
& x_{1}\left(n T^{+}\right)=\left(1-p_{2}\right) x_{1}(n T)+q \\
& \triangleq \Theta_{1}\left(x_{1}(n T), x_{2}(n T)\right), \\
& x_{2}\left(n T^{+}\right)=\left(1-p_{1}\right) x_{2}(n T) \\
& \triangleq \Theta_{2}\left(x_{1}(n T), x_{2}(n T)\right), \\
& t=n T .
\end{aligned}
$$

All notations used in this section are the same as those in [30]. Let $\Phi$ be the flow associated to (53); we have $x(t)=$ $\Phi\left(t, x_{0}\right), 0<t \leq T$, where $x(t)=\left(x_{1}(t), x_{2}(t)\right), x_{0}=x\left(0^{+}\right)$,

$$
d_{0}^{\prime}=1-\left(\frac{\partial \Theta_{2}}{\partial x_{2}} \frac{\partial \Phi_{2}}{\partial x_{2}}\right)\left(T_{0}, x_{0}\right)
$$

where $T_{0}$ is the root of $d_{0}^{\prime}=0$,

$$
\begin{gathered}
a_{0}^{\prime}=1-\left(\frac{\partial \Theta_{1}}{\partial x_{1}} \frac{\partial \Phi_{1}}{\partial x_{1}}\right)\left(T_{0}, x_{0}\right), \\
b_{0}^{\prime}=-\left(\frac{\partial \Theta_{1}}{\partial x_{1}} \frac{\partial \Phi_{1}}{\partial x_{2}}+\frac{\partial \Theta_{1}}{\partial x_{2}} \frac{\partial \Phi_{2}}{\partial x_{2}}\right)\left(T_{0}, x_{0}\right), \\
\frac{\partial \Phi_{1}\left(t, x_{0}\right)}{\partial x_{1}}=\exp \left(\int_{0}^{t} \frac{\partial F_{1}(\xi(r))}{\partial x_{1}} \mathrm{~d} r\right), \\
\frac{\partial \Phi_{2}\left(t, x_{0}\right)}{\partial x_{2}}=\exp \left(\int_{0}^{t} \frac{\partial F_{2}(\xi(r))}{\partial x_{2}} \mathrm{~d} r\right), \\
\frac{\partial \Phi_{1}\left(t, x_{0}\right)}{\partial x_{2}}=\int_{0}^{t} \exp \left(\int_{u}^{t} \frac{\partial F_{1}(\xi(r))}{\partial x_{1}} \mathrm{~d} r\right)\left(\frac{\partial F_{1}(\xi(u))}{\partial x_{2}}\right) \\
\frac{\partial^{2} \Phi_{2}\left(t, x_{0}\right)}{\partial x_{1} \partial x_{2}}=\int_{0}^{t} \exp \left(\int_{u}^{u} \frac{\partial F_{2}(\xi(r))}{\partial x_{2}} \mathrm{~d} r\right) \mathrm{d} u, \\
\times \exp \left(\int_{0}^{u} \frac{\partial F_{2}(\xi(r))}{\partial x_{2}} \mathrm{~d} r\right)\left(\frac{\partial^{2} F_{1}(\xi(u))}{\partial x_{1} \partial x_{2}}\right) \\
\mathrm{d} u,
\end{gathered}
$$




$$
\begin{aligned}
\frac{\partial^{2} \Phi_{2}\left(t, x_{0}\right)}{\partial x_{2}^{2}}= & \int_{0}^{t} \exp \left(\int_{u}^{t} \frac{\partial F_{2}(\xi(r))}{\partial x_{2}} \mathrm{~d} r\right)\left(\frac{\partial^{2} F_{2}(\xi(u))}{\partial x_{2}^{2}}\right) \\
& \times \exp \left(\int_{0}^{u} \frac{\partial F_{2}(\xi(r))}{\partial x_{2}} \mathrm{~d} r\right) \mathrm{d} u \\
+ & \int_{0}^{t} \exp \left(\int_{u}^{t} \frac{\partial F_{2}(\xi(r))}{\partial x_{2}} \mathrm{~d} r\right)\left(\frac{\partial^{2} F_{2}(\xi(u))}{\partial x_{1} \partial x_{2}}\right) \\
& \times \int_{0}^{u} \exp \left(\int_{p}^{u} \frac{\partial F_{1}(\xi(r))}{\partial x_{1}} \mathrm{~d} r\right)\left(\frac{\partial F_{1}(\xi(u))}{\partial x_{2}}\right) \\
& \times \exp \left(\int_{0}^{p} \frac{\partial F_{2}(\xi(r))}{\partial x_{2}} \mathrm{~d} r\right) \mathrm{d} p \mathrm{~d} u,
\end{aligned}
$$$$
\frac{\partial^{2} \Phi_{2}\left(t, x_{0}\right)}{\partial \bar{T} \partial x_{2}}=\frac{\partial F_{2}(\xi(t))}{\partial x_{2}} \exp \left(\int_{0}^{t} \frac{\partial F_{2}(\xi(r))}{\partial x_{2}} \mathrm{~d} r\right)
$$$$
\frac{\partial^{2} \Phi_{1}\left(T_{0}, x_{0}\right)}{\partial T}=y^{\prime}\left(T_{0}\right),
$$$$
B=-\frac{\partial^{2} \Theta_{2}}{\partial x_{1} \partial x_{2}}\left(\frac{\partial \Phi_{1}\left(T_{0}, X_{0}\right)}{\partial \bar{T}}\right.
$$$$
\left.+\frac{\partial \Phi_{1}\left(T_{0}, X_{0}\right)}{\partial x_{1}} \frac{1}{a_{0}^{\prime}} \frac{\partial \Theta_{1}}{\partial x_{1}} \frac{\partial \Phi_{1}\left(T_{0}, X_{0}\right)}{\partial \bar{T}}\right)
$$$$
\times \frac{\partial \Phi_{2}\left(T_{0}, X_{0}\right)}{\partial x_{2}}-\frac{\partial \Theta_{2}}{\partial x_{2}}\left(\frac{\partial^{2} \Phi_{2}\left(t, X_{0}\right)}{\partial \bar{T} \partial x_{2}}+\frac{\partial^{2} \Phi_{2}\left(t, X_{0}\right)}{\partial x_{1} \partial x_{2}}\right.
$$$$
\left.\times \frac{1}{a_{0}^{\prime}} \frac{\partial \Theta_{1}}{\partial x_{1}} \frac{\partial \Phi_{1}\left(T_{0}, X_{0}\right)}{\partial \bar{T}}\right),
$$$$
C=-2 \frac{\partial^{2} \Theta_{2}}{\partial x_{1} \partial x_{2}}\left(-\frac{b_{0}^{\prime}}{a_{0}^{\prime}} \frac{\partial \Phi_{1}\left(T_{0}, X_{0}\right)}{\partial x_{1}}+\frac{\partial \Phi_{1}\left(T_{0}, X_{0}\right)}{\partial x_{2}}\right)
$$$$
\times \frac{\partial \Phi_{2}\left(T_{0}, X_{0}\right)}{\partial x_{2}}-\frac{\partial^{2} \Theta_{2}}{\partial x_{2}^{2}}\left(\frac{\partial \Phi_{2}\left(T_{0}, X_{0}\right)}{\partial x_{2}}\right)^{2}
$$$$
+2 \frac{\partial \Theta_{2}}{\partial x_{2}} \frac{b_{0}^{\prime}}{a_{0}^{\prime}} \frac{\partial^{2} \Phi_{2}\left(T_{0}, X_{0}\right)}{\partial x_{1} \partial x_{2}}-\frac{\partial \Theta_{2}}{\partial x_{2}} \frac{\partial^{2} \Phi_{2}\left(T_{0}, X_{0}\right)}{\partial x_{2}^{2}},
$$

where $\xi(t)=\left(y^{*}(t), 0\right)$.

Lemma 11 (see [30]). If $\left|1-a_{0}^{\prime}\right|<1$ and $d_{0}^{\prime}=0$, then one has the following.

(a) If $B C \neq 0$, then one has a bifurcation. Moreover, one has a bifurcation of a nontrivial periodic solution of (53) if $B C<0$ and a subcritical case if $B C>0$.

(b) If $B C=0$, then one has an undetermined case.
In order to apply Lemma 11, we compute the following:

$$
\begin{aligned}
d_{0}^{\prime} & =1-\left(1-p_{1}\right) \exp \left(\int_{0}^{T}\left(a-\alpha y^{*}(r)\right) \mathrm{d} r\right) \\
& =1-\left(1-p_{1}\right) \exp \left[a T_{0}-\frac{\alpha q(1-\exp (-d T))}{d\left[1-\left(1-p_{2}\right) \exp (-d T)\right]}\right] .
\end{aligned}
$$

If $d_{0}^{\prime}=0$, this corresponds to $T_{0}$ satisfying

$$
a T_{0}=\frac{\alpha q\left(1-\exp \left(-d T_{0}\right)\right)}{d\left[1-\left(1-p_{2}\right) \exp \left(-d T_{0}\right)\right]}+\ln \left(\frac{1}{1-p_{1}}\right) .
$$

Further, we can get

$$
\begin{gathered}
a_{0}^{\prime}=1-\exp \left(-d T_{0}\right)>0, \\
b_{0}^{\prime}=-k \alpha\left(1-p_{2}\right) \exp \left(\int_{0}^{T_{0}} y^{*}(r) \mathrm{d} r\right)<0, \\
\frac{\partial \Phi_{1}\left(T_{0}, x_{0}\right)}{\partial x_{1}}=\exp \left(-d T_{0}\right)>0, \\
\frac{\partial \Phi_{2}\left(T_{0}, x_{0}\right)}{\partial x_{2}}=\exp \left[a T_{0}-\frac{\alpha q(1-\exp (-d T))}{d\left[1-\left(1-p_{2}\right) \exp (-d T)\right]}\right] \\
=\frac{1}{1-p_{1}}>0, \\
\frac{\partial \Phi_{1}\left(T_{0}, x_{0}\right)}{\partial x_{2}}=k \alpha \int_{0}^{T_{0}} \exp \left(-d\left(T_{0}-u\right)\right) y^{*}(u) \\
\frac{\partial^{2} \Phi_{2}\left(T_{0}, x_{0}\right)}{\partial \bar{T} \partial x_{2}}=\left(a-\frac{\alpha q\left(1-\exp \left(-d T_{0}\right)\right)}{d\left[1-\left(1-p_{2}\right) \exp \left(-d T_{0}\right)\right]}\right) \frac{1}{1-p_{1}}, \\
\frac{\left.\partial \Phi_{1}\left(a-\alpha y^{*}(r)\right) \mathrm{d} r\right) \mathrm{d} u>0,}{\partial x_{1} \partial x_{2}}=-\frac{k \alpha T_{0}}{1-p_{1}}<0, \\
\left.\frac{\partial T}{\partial}, x_{0}\right) \\
y^{\prime}\left(T_{0}\right)=-\frac{d q \exp \left(-d T_{0}\right)}{1-\left(1-p_{2}\right) \exp \left(-d T_{0}\right)}<0 .
\end{gathered}
$$

Note that

$$
\begin{gathered}
\frac{\partial^{2} F_{2}(\xi(u))}{\partial x_{2}^{2}}=-\left(2 b+\alpha y^{*}(u)\right)<0, \\
\frac{\partial^{2} F_{2}(\xi(u))}{\partial x_{1} \partial x_{2}}=-\alpha<0, \\
\frac{\partial F_{1}(\xi(u))}{\partial x_{2}}=k \alpha y^{*}(u)>0 ;
\end{gathered}
$$

then

$$
\frac{\partial^{2} \Phi_{2}\left(T_{0}, x_{0}\right)}{\partial x_{2}^{2}}<0
$$



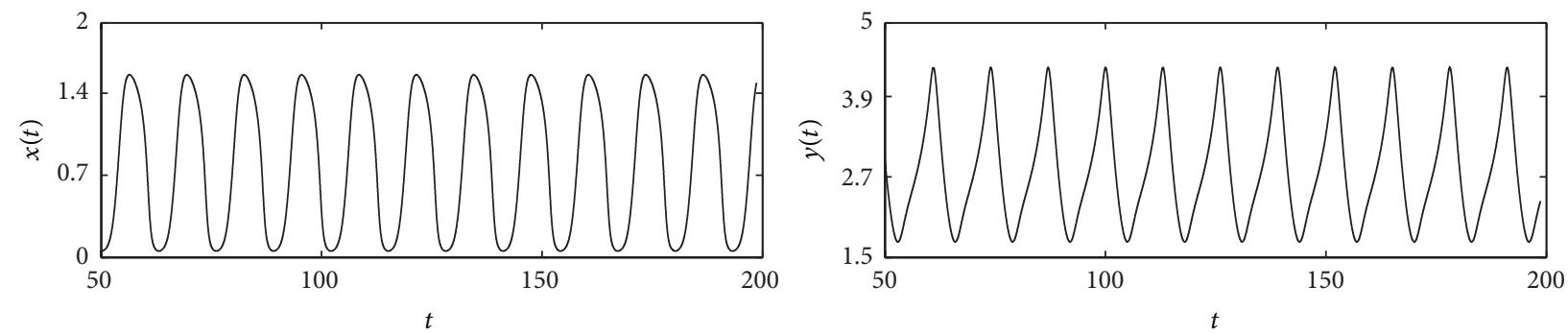

(a)
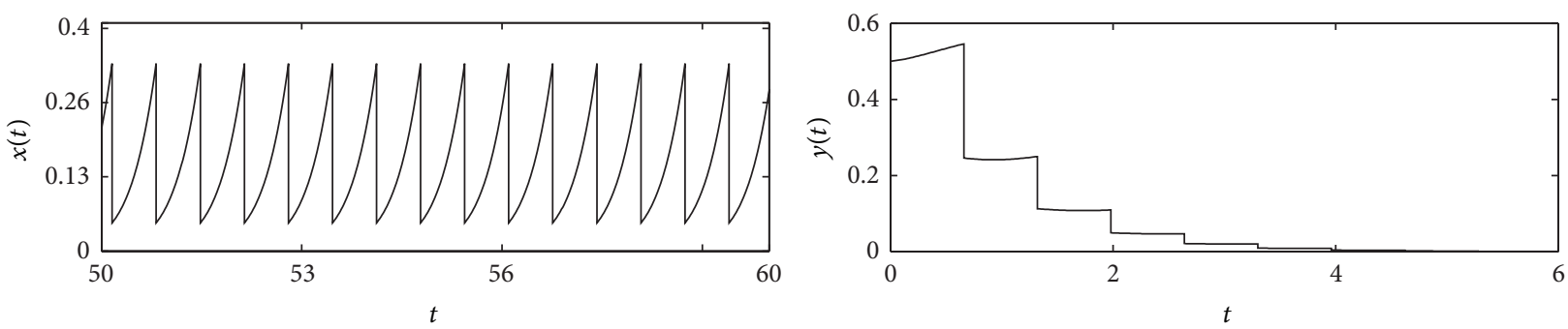

(b)
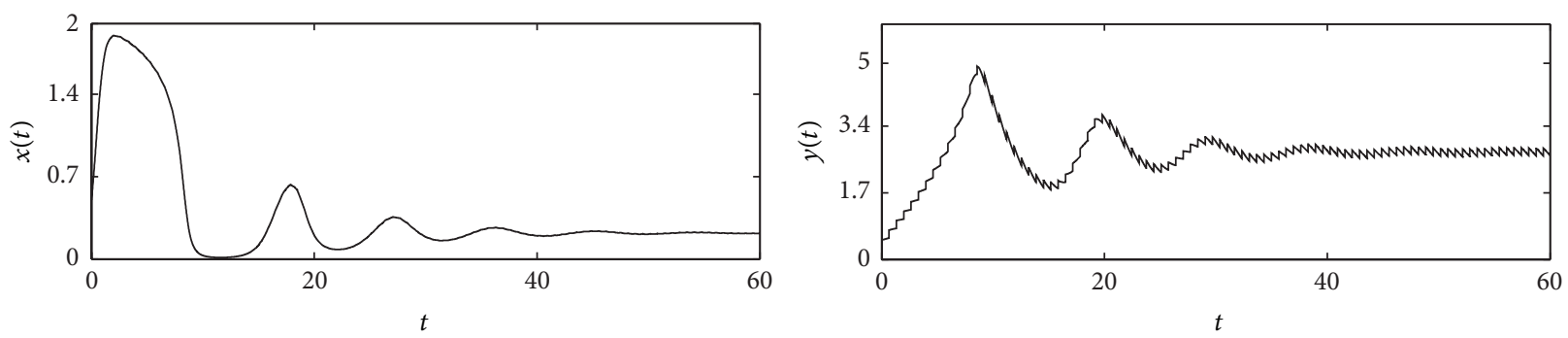

(c)
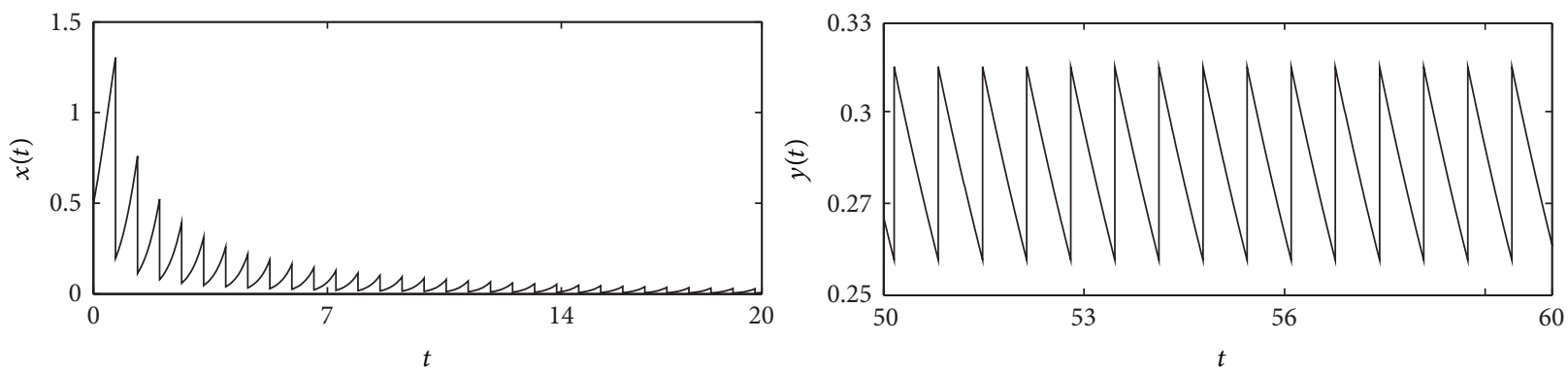

(d)

FIGURE 1: Time series of system (11) when $T=0.66<T_{\max } \approx 0.6776$. (a) $p_{1}=0, p_{2}=0$, and $q=0$, without taking any pest-management strategy; (b) $p_{1}=0.85, p_{2}=0.55$, and $q=0$, with spraying pesticide (or harvesting) only; (c) $p_{1}=0, p_{2}=0$, and $q=0.2$, with releasing of predator (natural enemy) only; (d) $p_{1}=0.85, p_{2}=0.55$, and $q=0.2$, with taking integrated pest-management strategy.

Since

$$
\frac{\partial \Theta_{1}}{\partial x_{2}}=\frac{\partial \Theta_{2}}{\partial x_{1}}=0, \quad \frac{\partial \Theta_{1}}{\partial x_{1}}=1-p_{2}, \quad \frac{\partial \Theta_{2}}{\partial x_{2}}=1-p_{1},
$$

it is easy to verify that $C>0$ and

$$
\begin{array}{r}
B=-\left[a-\frac{\alpha q \exp \left(-d T_{0}\right)}{d\left[1-\left(1-p_{2}\right) \exp \left(-d T_{0}\right)\right]}\right. \\
\left.+\frac{k \alpha d q T_{0}\left(1-p_{2}\right) \exp \left(-d T_{0}\right)}{\left[1-\left(1-p_{2}\right) \exp \left(-d T_{0}\right)\right]^{2}}\right] .
\end{array}
$$

In order to determine the $\operatorname{sign}$ of $B$, let

$$
f(t)=a-\frac{\alpha q \exp (-d t)}{1-\left(1-p_{2}\right) \exp \left(-d T_{0}\right)} .
$$

We have

$$
f^{\prime}(t)=\frac{\alpha q \exp (-d t)}{d\left[1-\left(1-p_{2}\right) \exp \left(-d T_{0}\right)\right]}>0 .
$$




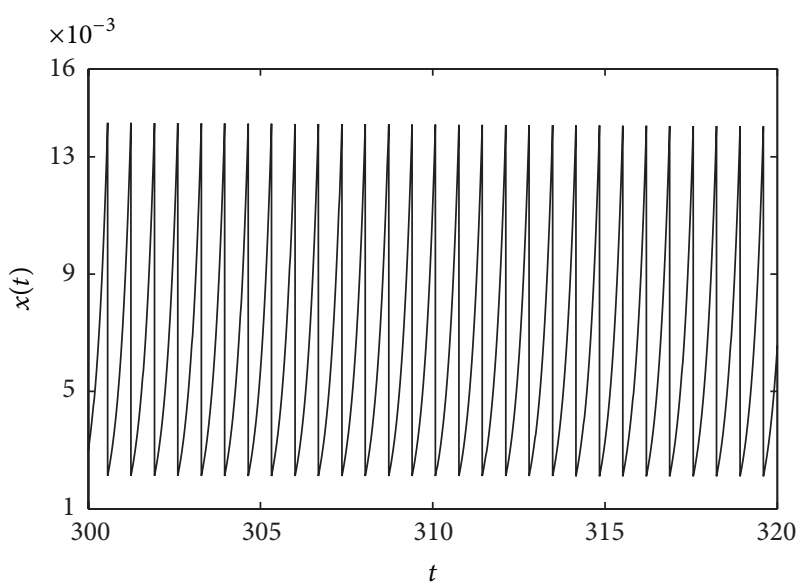

(a)

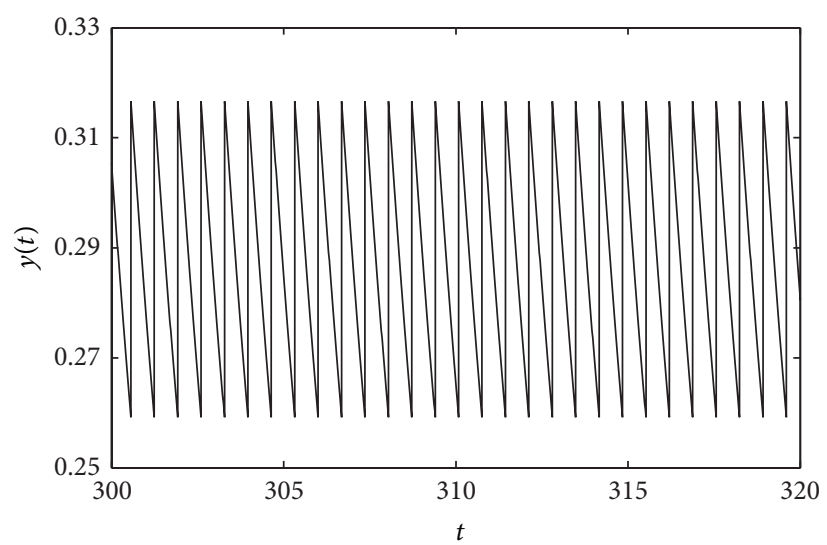

(b)

FIGURE 2: Time series of system (11) when $T=0.68>T_{\max } \approx 0.6776$; prey population $x$ and predator population $y$ coexist with periodic oscillations.
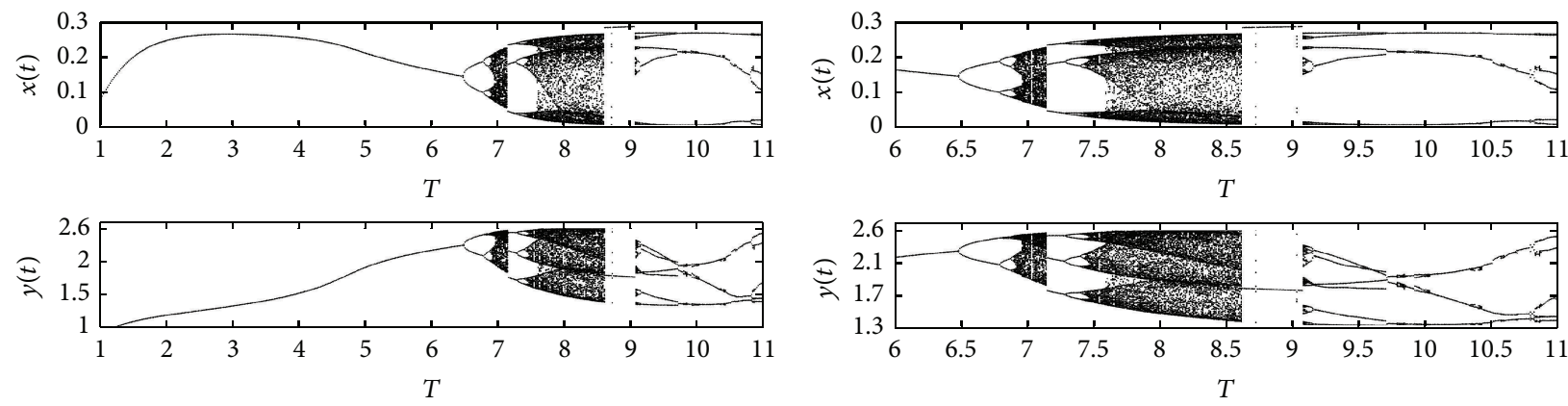

(a)

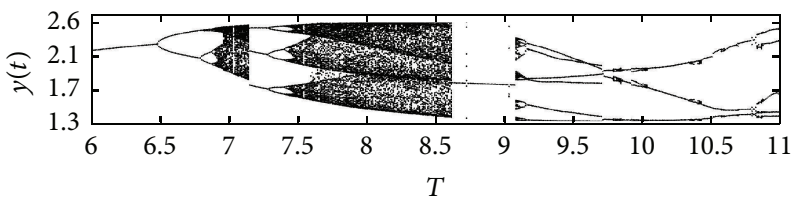

(b)

FIGURE 3: Bifurcation diagrams of system (11): (a) prey population $x$ and predator population $y$ with impulsive period T over [1, 11]; (b) prey population $x$ and predator population $y$ with impulsive period $T$ over $[6,11]$.

Thus, we can conclude that $f\left(T_{0}\right)>0$, since

$$
\begin{aligned}
\int_{0}^{T_{0}} f(t) \mathrm{d} t & =a T_{0}-\frac{\alpha q \exp (-d t)}{d\left[1-\left(1-p_{2}\right) \exp \left(-d T_{0}\right)\right]} \\
& =\ln \left(\frac{1}{1-p_{1}}\right) \geq 0,
\end{aligned}
$$

and $f(t)$ is strictly increasing. Therefore, we have $B<0$. In view of $T_{0}=T_{\max }$ and according to Lemma 11, we obtain the following result.

Theorem 12. System (11) has a positive periodic solution if T > $T_{0}$ and $T$ is close to $T_{0}$, where $T_{0}$ satisfies

$$
a T_{0}=\frac{\alpha q\left(1-\exp \left(-d T_{0}\right)\right)}{d\left[1-\left(1-p_{2}\right) \exp \left(-d T_{0}\right)\right]}+\ln \left(\frac{1}{1-p_{1}}\right),
$$

and the nontrivial periodic solution is supercritical case via bifurcation, which means that the positive periodic solution is stable.

\section{Numerical Analysis}

In this section, we will study the impulsive effect on system (11) and show that the impulsive perturbations cause complicated dynamical behavior for system (11). The influence of $T, q$, and $\beta$ may be documented by stroboscopically sampling one of the variables over a range of their values. Stroboscopic map is a special case of the Poincaré map for periodically forced system or periodically pulsed system. Fixing points of the stroboscopic map correspond to periodic solutions of system (11) having the same period as the pulsing term; periodic points of period $k$ about stroboscopic map correspond to entrained periodic solutions of system (11) having exactly $k$ times the period of the pulsing; invariant circles correspond to quasi-periodic solutions of system (11); system (11) possibly appear chaotic (or strange) attractors.

Example 13. Let $a=3.1, b=1.5, \alpha=1.05, k=0.85, n=$ $2.15, h=0.97, d=0.3, p_{1}=0.85, p_{2}=0.55$, and $q=0.2$ with initial value $X(0)=(0.5,0.5)$. 


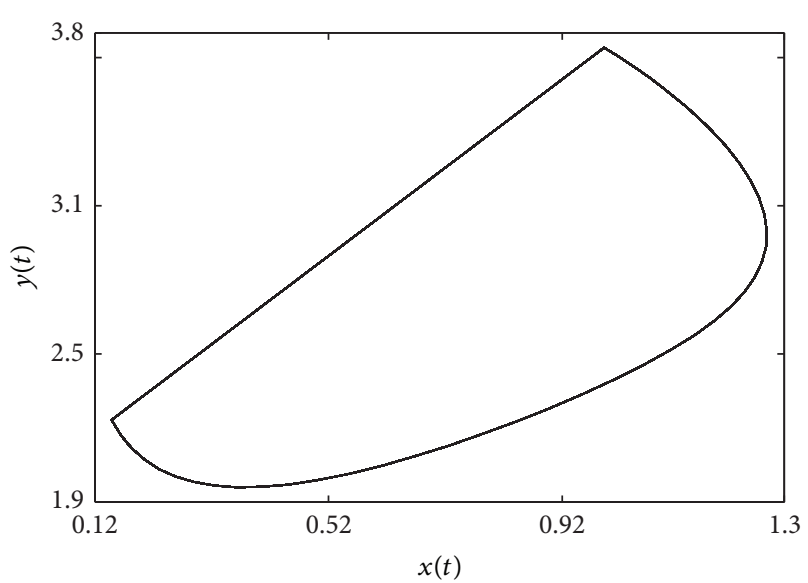

(a)

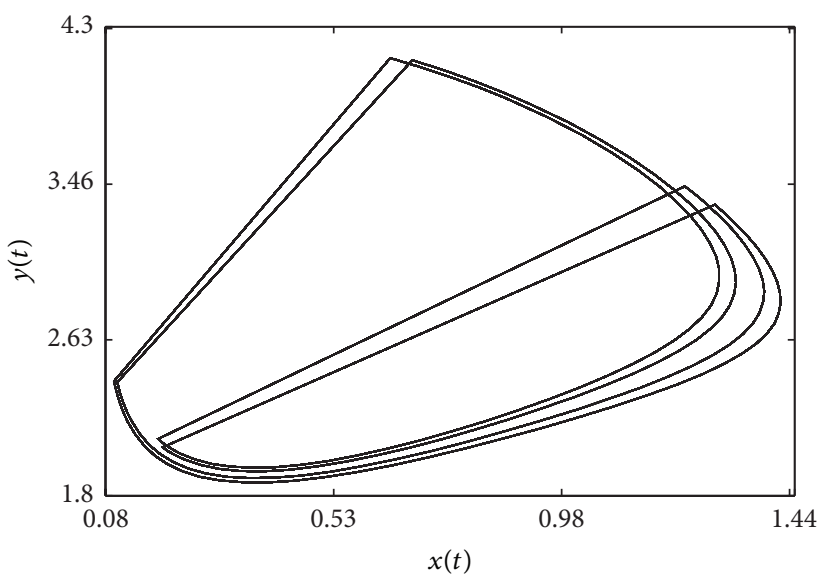

(c)

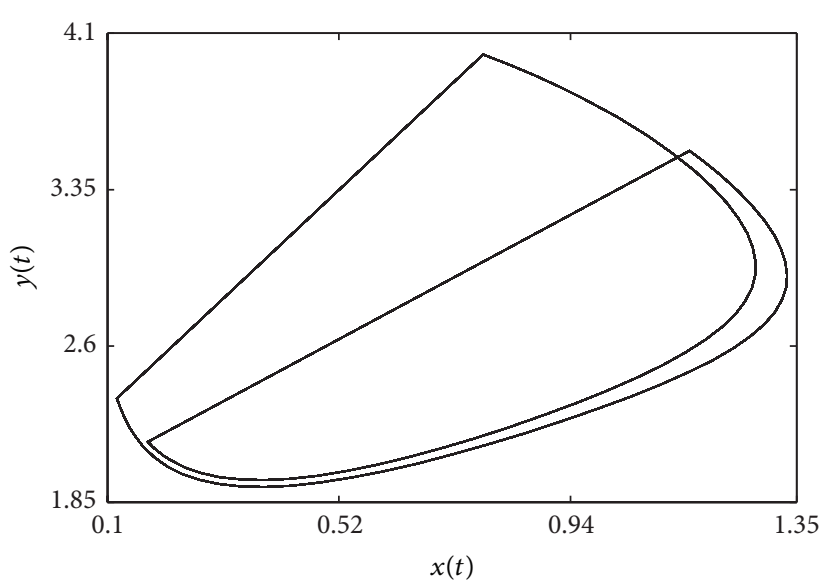

(b)

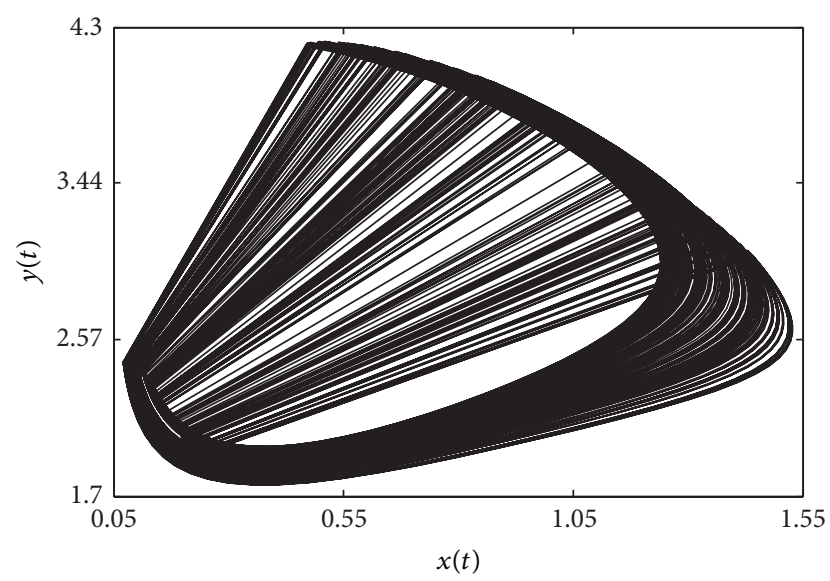

(d)

FIgURE 4: Period doubling bifurcation leads to chaos: $T, 2 T$, and $4 T$ periodic solutions and chaos when $T=6.4,6.6,6.8$, and 7.0 , respectively.

From Remark 10, large numbers of preys (pest) could coexist with predators (natural enemy) with periodic oscillations, if we are not taking pest-management strategy $\left(p_{1}=\right.$ $0, p_{2}=0, q=0$ ) (Figure $\left.1(\mathrm{a})\right) \cdot q=0, p_{1}=$ 0.85 , and $p_{2}=0.55$; that is, there are periodic spraying pesticide (or harvesting) only, without releasing of predator (natural enemy), large numbers of preys (pest) coexist with periodic oscillation, but predators (natural enemy) rapidly decrease to zero when $T=0.66<T_{\max }$ (Figure 1(b)). $p_{1}=$ $0, p_{2}=0, q=0.2$; that is, there is periodic releasing of predator (natural enemy) only; without spraying pesticide (or harvesting), a few of preys (pest) coexist with predators (natural enemy) when $T=0.66<T_{\max }$ (Figure 1(c)). We cannot make the prey population $x(t)$ eradicate when $T=$ 0.66 . From Theorem 6, we know that the prey-eradication periodic solution is asymptotically stable provided that $T<$ $T_{\max } \approx 0.6776$. A typical prey-eradication periodic solution of the system (11) is shown in Figure 1(d), where we observe how the variable $y(t)$ oscillates in a stable cycle. In contrast, the prey population $x(t)$ rapidly decreases to zero when $T=0.66<T_{\max } \approx 0.6776$. Hence, the integrated pest management (IPM) is better than the classic methods (such as biological control or chemical control).
According to Theorem 12, if the impulsive periodic $T>$ $T_{\max }$ and is close to $T_{\max }$, the prey eradication solution becomes unstable, there is a supercritical bifurcation, then the prey and predator can coexist on a stable positive periodic solution when $T=0.68>T_{\max } \approx 0.6776$ (Figure 2). Therefore, in order to control the pest populations, we would choose an appropriate impulsive periodic $T . T>T_{\max }$ and close to $T_{\max }$ would be a better one.

Let $q=0.55$ and fix other parameter sets of values; we have displayed bifurcation diagrams for the pest population $x$ and the predator population $y$ for impulsive period $T$ over $[1,11]$ and $[6,11]$. We find that by increasing the impulsive period $T$, system (11) undergoes a process of period-doubling cascade $\rightarrow$ chaos $\rightarrow$ crisis and high-order periodic oscillations (Figure 3). When $T$ increases from 6 to 7 , there is a cascade of period-doubling bifurcations leading to chaos (Figure 4). When $T=8.62$, the chaos suddenly disappears and a $T$-periodic solution appears, then the $T$ periodic solution abruptly disappears and the chaos abruptly appears again when $T=9.08$, these constituting several types of crises (Figure 5). However, when $T=8.62$ and $T=$ 9.08 , it appears that attractors are nonunique, coexistence of stranger attractor with $T$-periodic solution (Figure 6). 


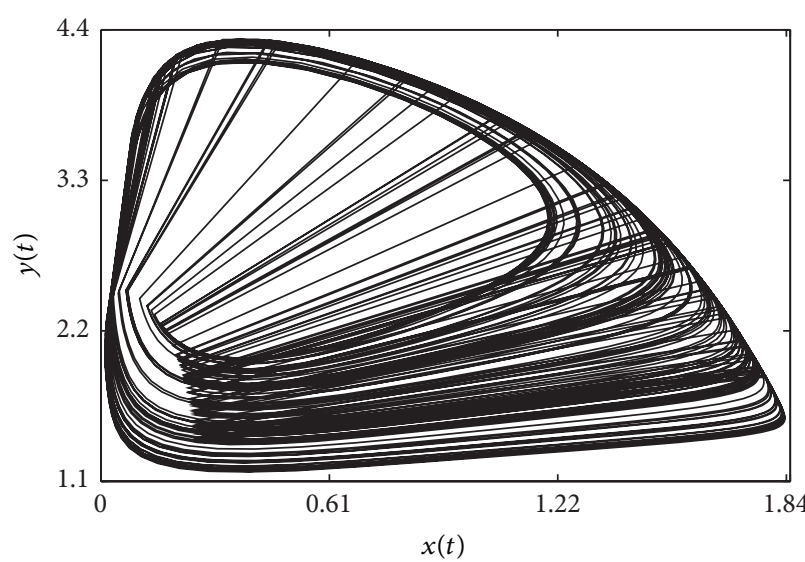

(a)

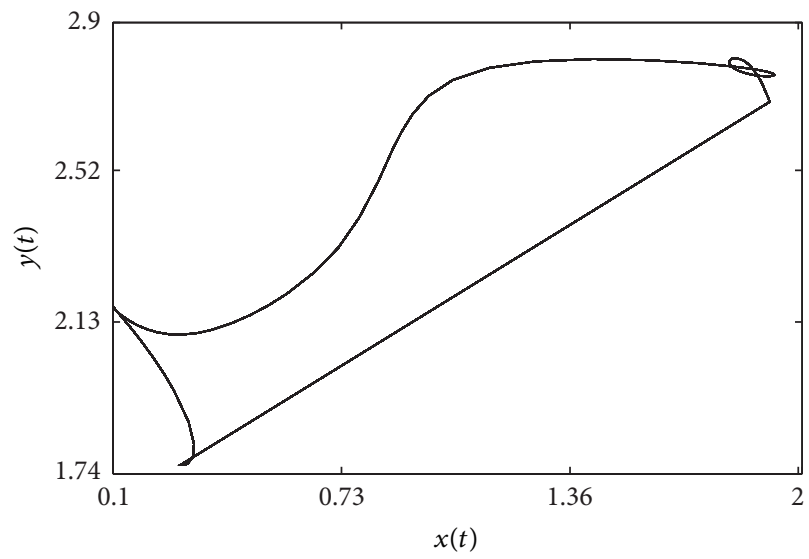

(c)

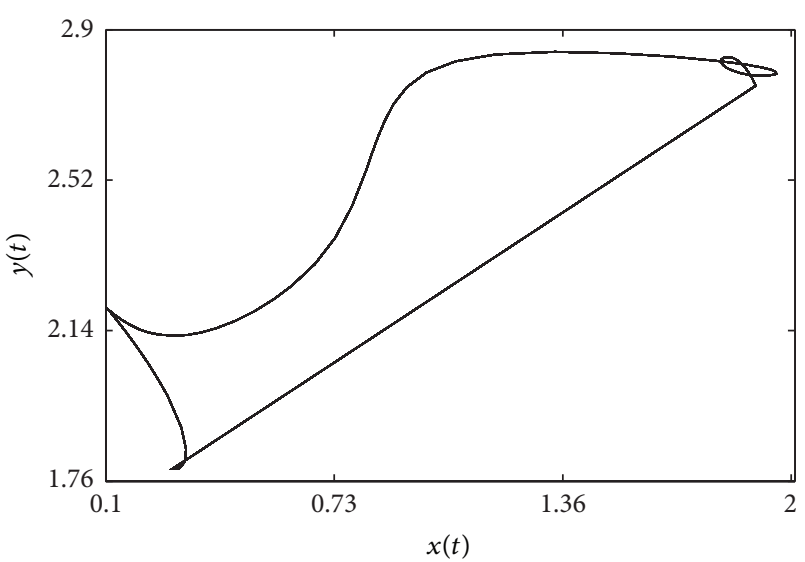

(b)

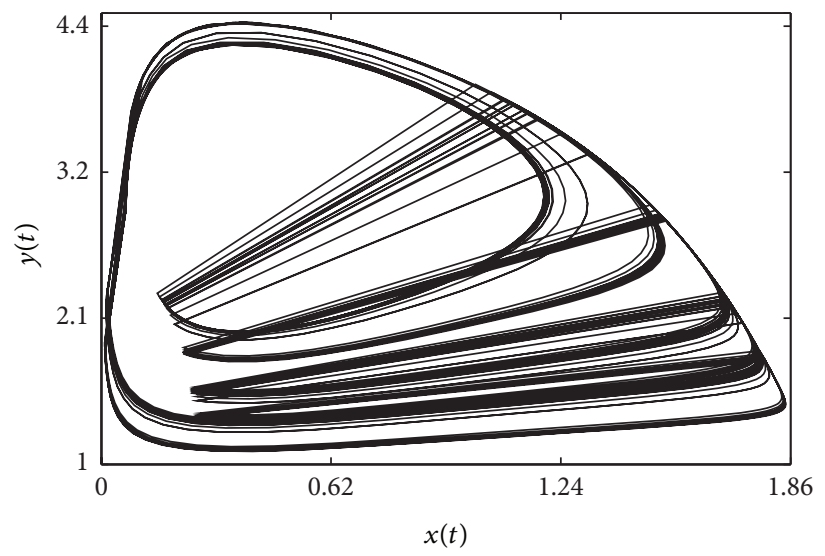

(d)

Figure 5: Crises are shown. There is a crisis that the chaos suddenly disappears when $T=8.61,8.62$, and there is a crisis that the chaos suddenly appears when $T=9.07,9.08$.

Obviously, which one of the attractors is reached depends on the initial values.

Example 14. Let $a=3.1, b=1.5, \alpha=1.05, k=0.85, n=$ $2.15, h=0.97, d=0.3, p_{1}=0.85, p_{2}=0.55$, and $T=8$ with initial value $X(0)=(0.5,0.5)$. We investigate the effect of $q$ on the system (11). Figure 7 showed bifurcation diagrams obtained by stroboscopically sampling the pest population $x$ and the predator population $y$ for $q$ over $[0.1,3.1]$. The resulting bifurcation diagrams clearly showed that system (11) has rich dynamics, including period-doubling bifurcation, period-halving bifurcation, and chaos. When $q$ increases from 0.9 to 2.2 , there is a period-halving bifurcation leading to a $T$-periodic solution (Figure 8).

Example 15. Let $a=3.1, b=1.5, \alpha=1.05, k=0.85, n=$ $2.15, h=0.97, d=0.3, p_{1}=0.85, p_{2}=0.55, q=$ 1.2 , and $T=8$ with initial value $X(0)=(0.5,0.5)$. We consider the effect of $\beta$ on the system (11). The resulting bifurcation diagrams (Figure 9), the pest population $x$, and the predator population $y$ for $\beta$ over $[2.0,6.0]$ clearly showed that system (11) has complex dynamics, such as period-doubling bifurcation, high-order periodic oscillation, and chaos. In
Figure 10, the typical high-order oscillation of system (11) is shown: $7 T, 12 T, 17 T$, and $3 T$ periodic solutions when $\beta=$ $2.8,3.05,3.4$, and 5.5, respectively. Further, Figure 11 shown the maximin and mean amount of prey population $x$ and predator population $y$ of system (11) with $\beta$ over [2.0, 5.5].

From bifurcation diagrams in Figures 3, 7, and 9, we can easily see that the dynamical behavior of these three cases is very complicated, which includes (1) high-order quasi-periodic and periodic oscillations, (2) period-doubling bifurcation, (3) period-halving bifurcations, (4) nonunique dynamics (meaning that several attractors coexist), and (5) cries (the phenomenon of "crisis" in chaotic attractors can suddenly appear or disappear, or change size discontinuously as a parameter smoothly varies).

\section{Conclusion}

In this paper, we have investigated a predator-prey system with generalized group defense and concerning impulsive control strategy for pest control in detail. We have shown that there exists an asymptotically stable pest-eradication periodic 


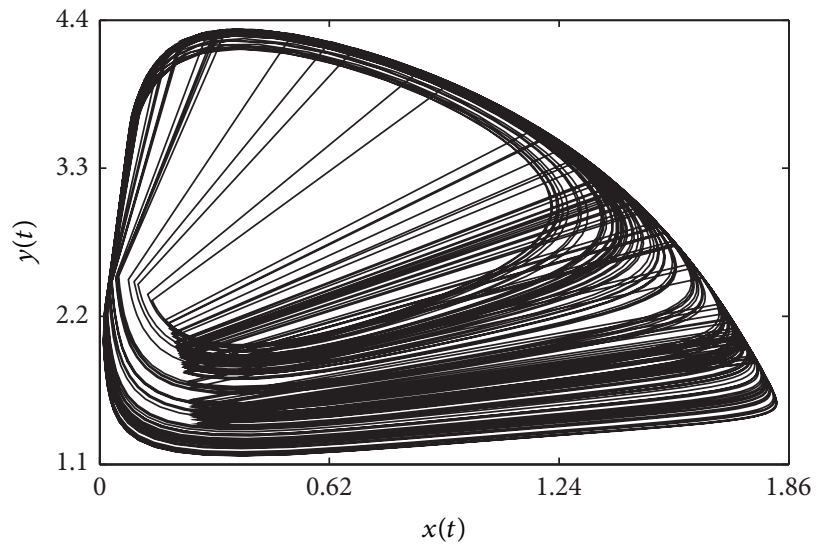

(a)

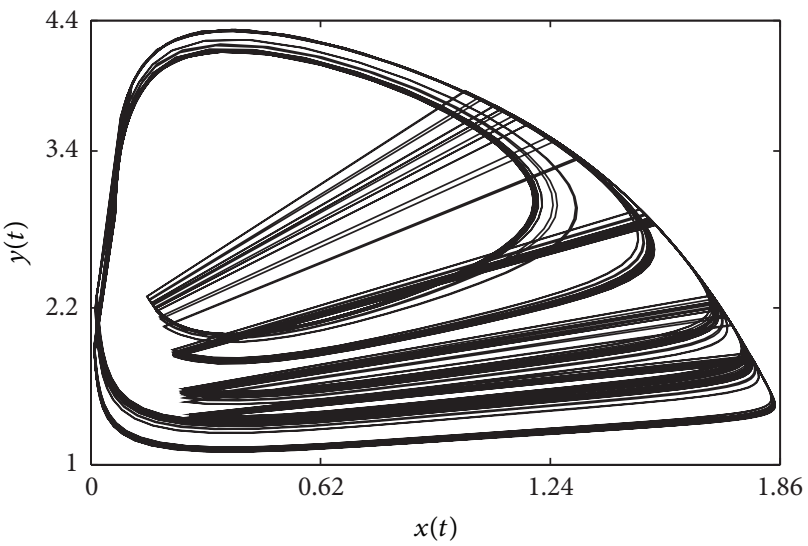

(c)

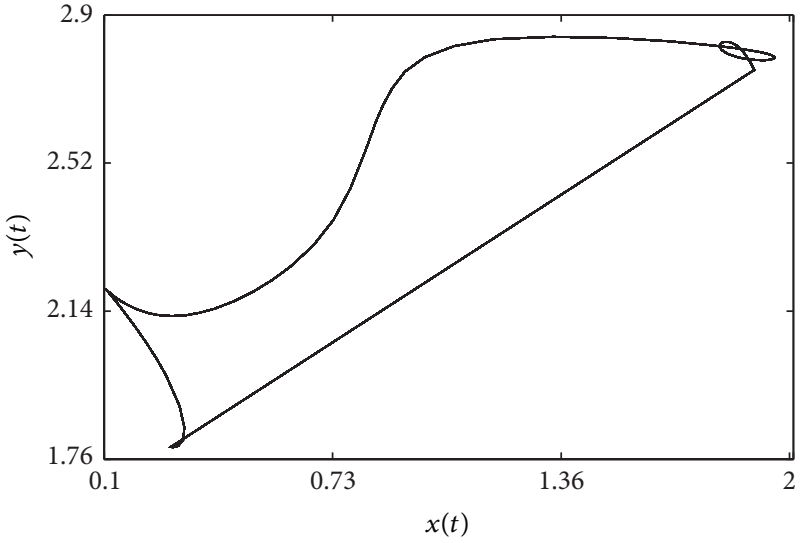

(b)

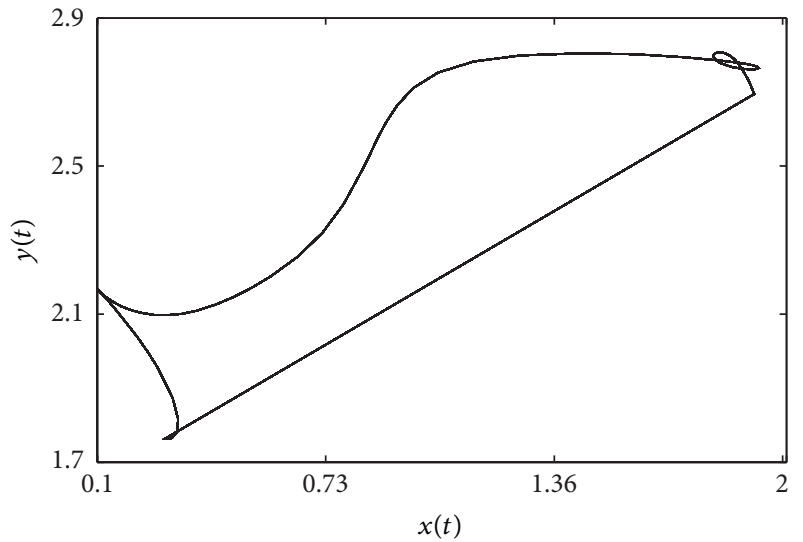

(d)

FIGURE 6: Different attractors' coexistence: solution with initial value $X(0)=(0.5,2.5)$ will finally tend to strange attractor, solution with $X(0)=(0.6,2.5)$ will finally tend to $T$ periodic solution when $T=8.62$, solution with initial value $X(0)=(1.1,2.5)$ will finally tend to strange attractor, and solution with $X(0)=(1.2,2.5)$ will finally tend to $T$ periodic solution when $T=9.08$, respectively.

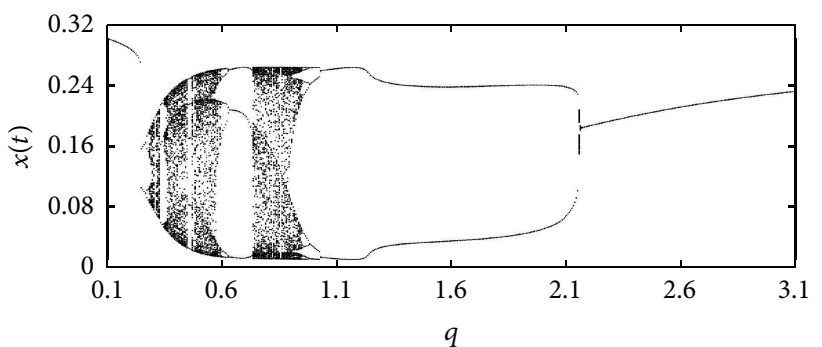

(a)

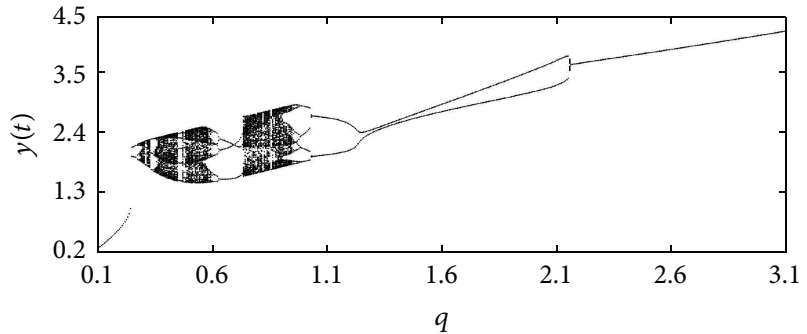

(b)

FIGURE 7: Bifurcation diagrams of system (11): prey population $x$ and predator population $y$ with $q$ over $[0.1,3.1]$.

solution if the impulsive period is less than the critical value $T_{\max }$. If we choose our impulsive control strategy, in order to drive the pest to extinction, we can determine the impulsive period $T$ according to the effect of the chemical pesticides on the populations and the cost of releasing natural enemies such that $T<T_{\max }$.

But, in a real world, complete eradication of pest populations is generally not possible, nor is it biologically or economically desirable. A good-pest control program should reduce pest population to levels acceptable to the public. When $T>T_{\max }$, the stability of the pest-eradication periodic solution is lost, system (11) is permanent, and there exists a nontrivial periodic solution when $T$ is close to $T_{\max }$. The smaller the period, the fewer the pests. Therefore, we can control the pest population below some economic threshold (ET is defined as the pest population level that produces 


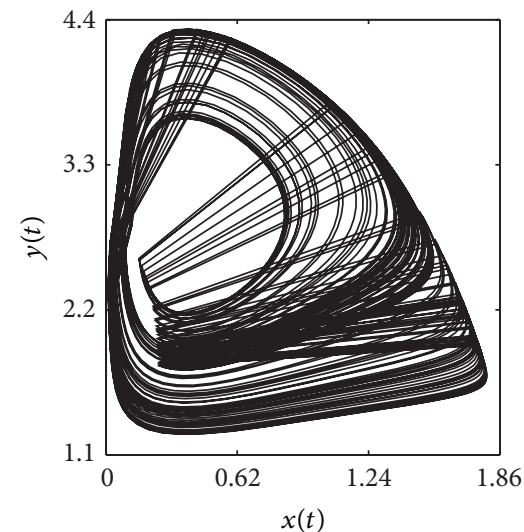

(a)

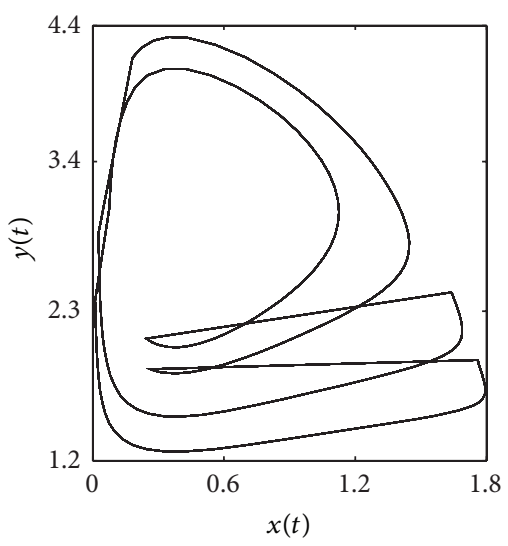

(d)

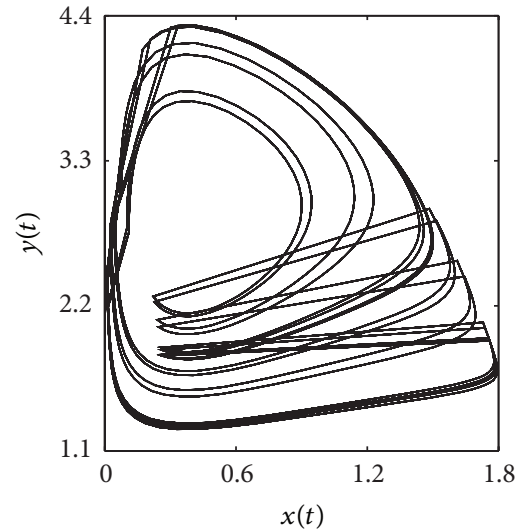

(b)

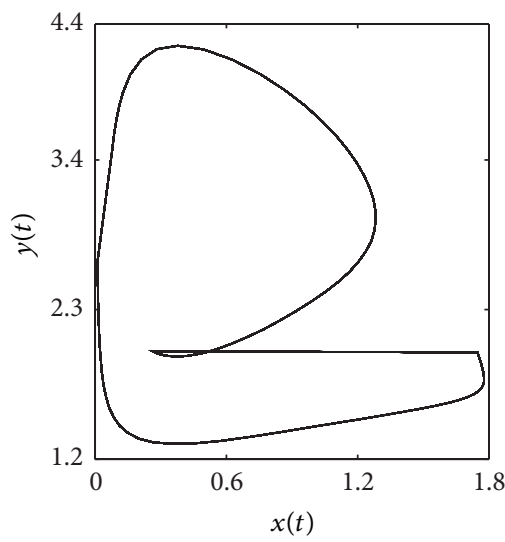

(e)

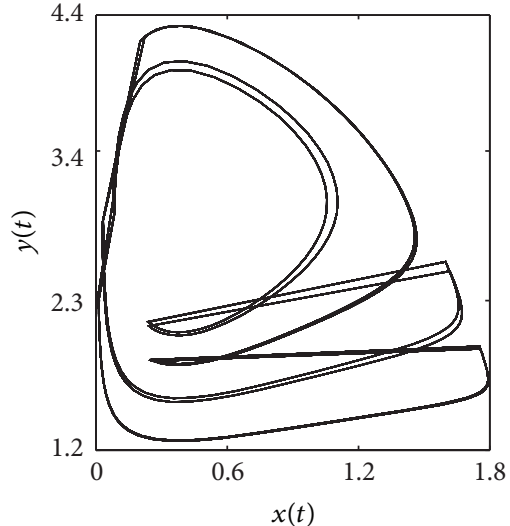

(c)

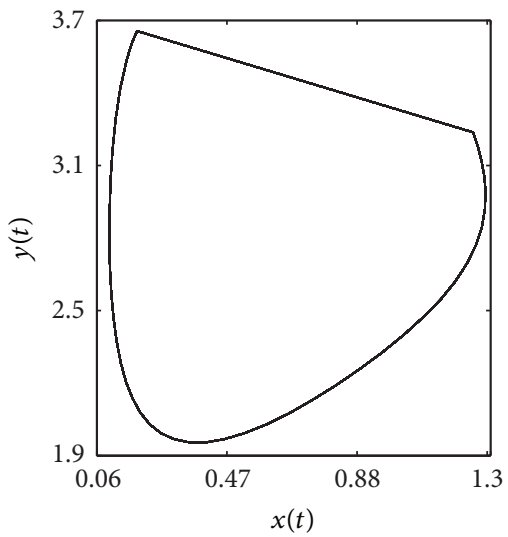

(f)

FIGURE 8: Period-halving bifurcation leads to a $T$-periodic solution of system (11): chaos and 16T, 8T, 4T, $2 T$, and $T$ periodic solutions when $q=0.9,0.95,0.98,1.0,1.1$, and 2.2 , respectively.

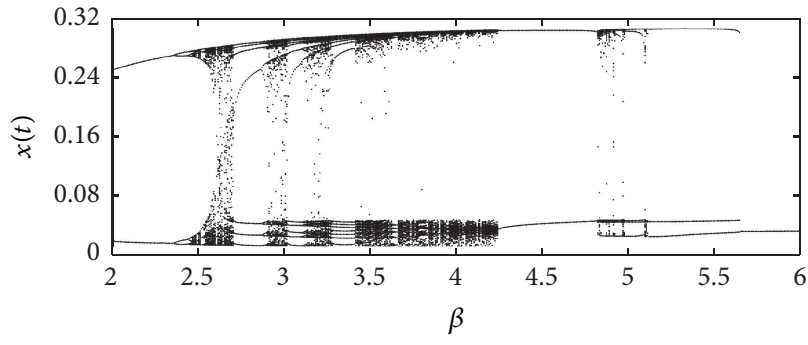

(a)

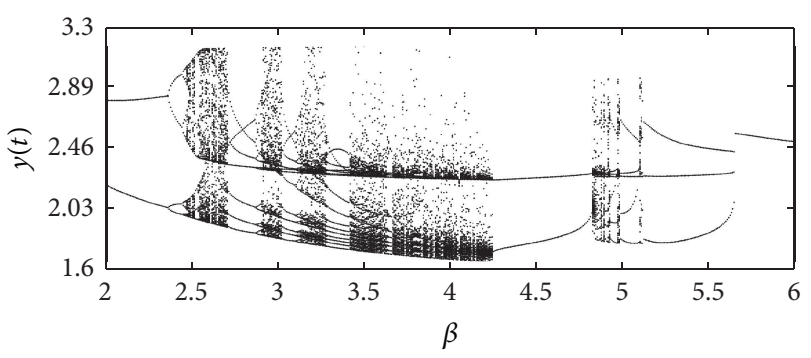

(b)

FIGURE 9: Bifurcation diagrams of system (11): prey population $x$ and predator population $y$ with $\beta$ over [2.0,6.0].

damage equal to the costs of preventing damage) by choosing appropriate impulsive period $T$ and the number of mature predator released $q$, according to the degree of antipredator behavior and group defense $\beta$, making an integrated pestmanagement strategy every period $T$. Then, the periodic releasing of natural enemies and spraying pesticides change the properties of the system without impulses and our results suggest an effective approach in the pest control.
Numerical results show that system (11) can take on various kinds of periodic fluctuations and several types of attractor coexistence and is dominated by high-order periodic oscillations, quasi-periodic oscillations, and chaotic oscillations. These results imply that the presence of pulses destroys equilibria, initiates multiple attractors, quasi-periodic oscillations, and chaos, and makes the dynamical behaviors more complex. 


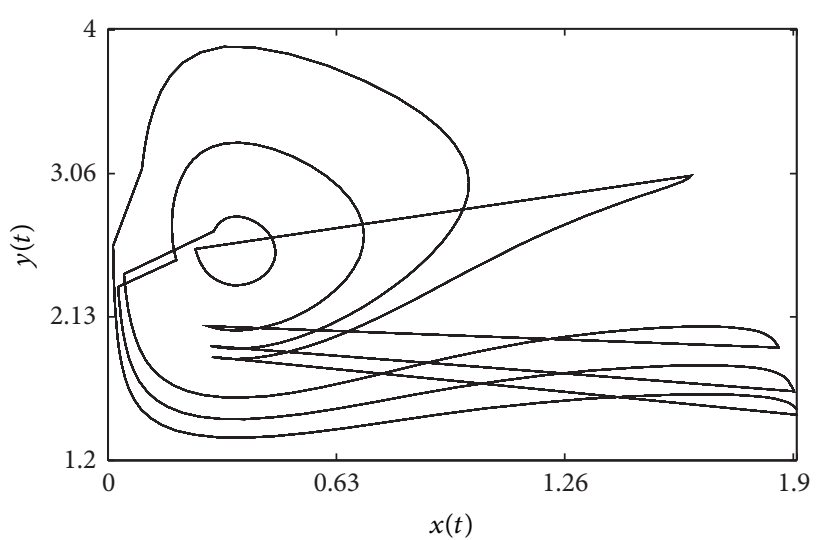

(a)

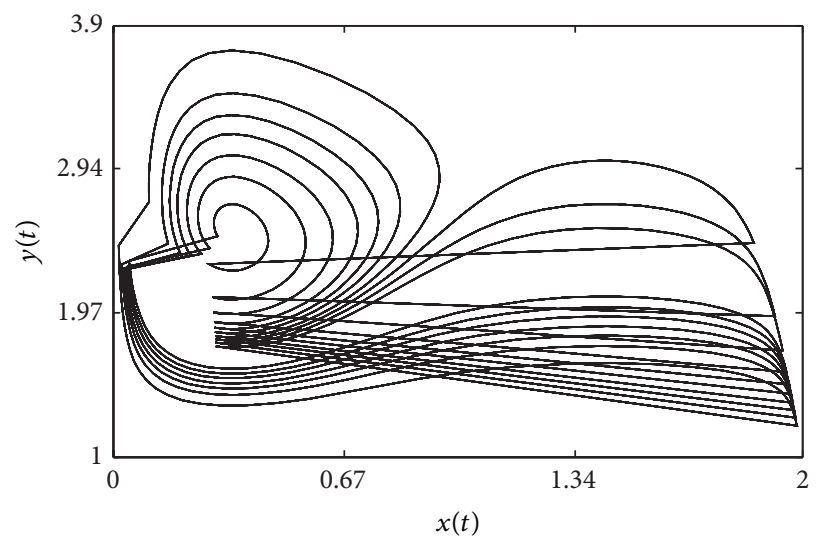

(c)

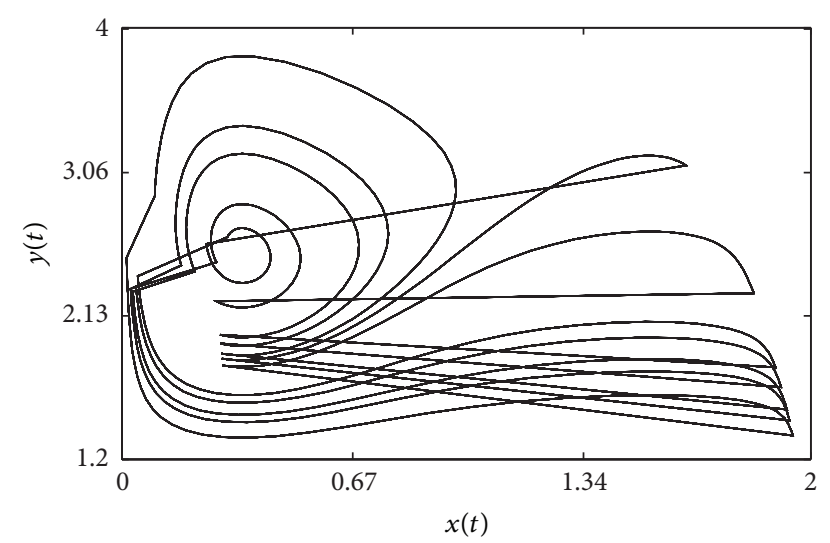

(b)

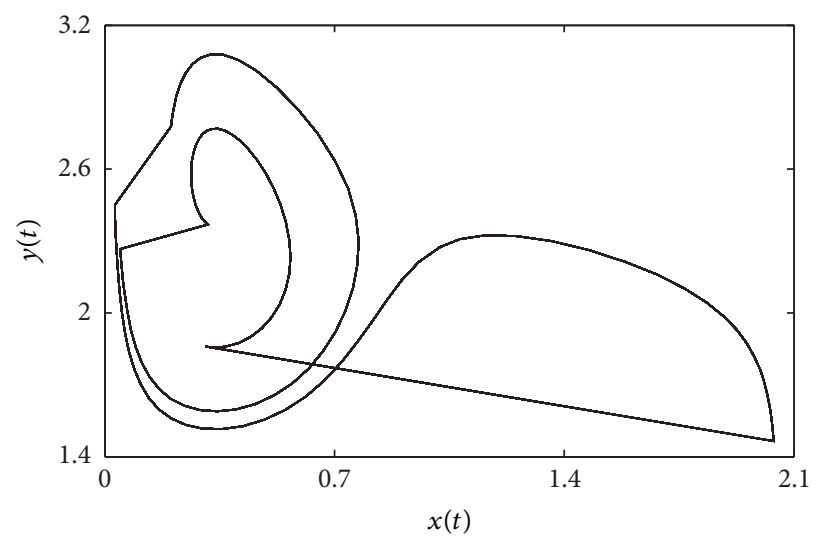

(d)

FIGURE 10: High-order oscillations of system (11): $7 T, 12 T, 17 T$, and $3 T$ periodic solutions when $\beta=2.8,3.05$, 3.4, and 5.5 , respectively.

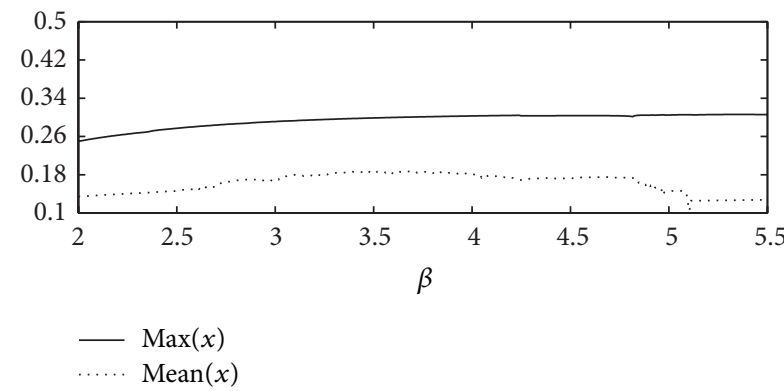

(a)

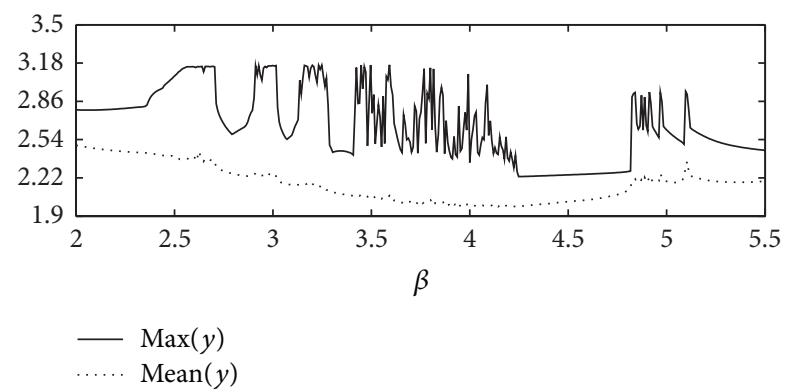

(b)

FIGURE 11: The maximin and mean amount of prey population $x$ and predator population $y$ of system (11) with $\beta$ over [2.0,5.5], respectively.

\section{Acknowledgment}

This work was supported by the Science and Research Project Foundation of Educational Department of Guizhou Province (no. 2010096).

\section{References}

[1] C. S. Holling, "The functional response of predator to prey density and its role in mimicry and population regulation," Memoirs of the Entomological Society of Canada, vol. 1, no. 45, pp. 1-60, 1965.
[2] H. I. Freedman, Deterministic Mathematical Models in Population Ecology, vol. 57 of Monograph Textbooks Pure and Applied Mathematics, Marcel Dekker, New York, NY, USA, 1980.

[3] V. S. Ivlev, Experimental Ecology of the Feeding of Fishes, Yale University Press, New Haven, Conn, USA, 1961.

[4] D. L. DeAngelis, R. A. Goldstein, and R. V. O’Neill, “A Model for trophic interaction," Ecology, vol. 56, pp. 881-892, 1975.

[5] J. R. Beddington, "Mutual interference between parasites or predators and its effect on searching efficiency," Journal of Animal Ecology, vol. 44, pp. 331-340, 1975.

[6] D. A. Miller, J. B. Grand, T. F. Fondell, and M. Anthony, "Predator functional response and prey survival: direct and indirect 
interactions affecting a marked prey population," Journal of Animal Ecology, vol. 75, no. 1, pp. 101-110, 2006.

[7] J. F. Andrews, "A mathematical model for the continuous culture of microorganisms utilizing inhibitory substrates," Biotechnology and Bioengineering, vol. 10, pp. 707-723, 1968.

[8] H. I. Freedman and S. G. Ruan, "Hopf bifurcation in threespecies food chain models with group defense," Mathematical Biosciences, vol. 111, no. 1, pp. 73-87, 1992.

[9] H. I. Freedman and G. S. K. Wolkowicz, "Predator-prey systems with group defence: the paradox of enrichment revisited," Bulletin of Mathematical Biology, vol. 48, no. 5-6, pp. 493-508, 1986.

[10] H. I. Freedman and H. S. Quan, "Interactions leading to persistence in predator-prey systems with group defence," Bulletin of Mathematical Biology, vol. 50, no. 5, pp. 517-530, 1988.

[11] D. M. Xiao and S. G. Ruan, "Codimension two bifurcations in a predator-prey system with group defense," International Journal of Bifurcation and Chaos in Applied Sciences and Engineering, vol. 11, no. 8, pp. 2123-2131, 2001.

[12] H. Ddumba, J. Y. T. Mugisha, J. W. Gonsalves, and G. I. H. Kerley, "The role of predator fertility and prey threshold bounds on the global and local dynamics of a predator-prey model with a prey out-flux dilution effect," Applied Mathematics and Computation, vol. 218, no. 18, pp. 9169-9186, 2012.

[13] S. W. Zhang, F. Y. Wang, and L. S. Chen, "A food chain model with impulsive perturbations and Holling IV functional response," Chaos, Solitons and Fractals, vol. 26, no. 3, pp. 855866, 2005.

[14] Z. L. Xiong, Y. Xue, and S. Y. Li, "A food chain system with Holling IV functional responses and impulsive effect," International Journal of Biomathematics, vol. 1, no. 3, pp. 361375, 2008.

[15] H. Baek, "A food chain system with Holling type IV functional response and impulsive perturbations," Computers \& Mathematics with Applications, vol. 60, no. 5, pp. 1152-1163, 2010.

[16] J. J. Jiao, X. Z. Meng, and L. S. Chen, "A stage-structured Holling mass defence predator-prey model with impulsive perturbations on predators," Applied Mathematics and Computation, vol. 189, no. 2, pp. 1448-1458, 2007.

[17] S. W. Zhang, D. J. Tan, and L. S. Chen, "Chaos in periodically forced Holling type IV predator-prey system with impulsive perturbations," Chaos, Solitons and Fractals, vol. 27, no. 4, pp. 980-990, 2006.

[18] Z. J. Liu and R. H. Tan, "Impulsive harvesting and stocking in a Monod-Haldane functional response predator-prey system," Chaos, Solitons and Fractals, vol. 34, no. 2, pp. 454-464, 2007.

[19] Q. Wang, B. X. Dai, and Y. M. Chen, "Multiple periodic solutions of an impulsive predator-prey model with Hollingtype IV functional response," Mathematical and Computer Modelling, vol. 49, no. 9-10, pp. 1829-1836, 2009.

[20] H. Baek and Y. Do, "Stability for a Holling type IV food chain system with impulsive perturbations," Kyungpook Mathematical Journal, vol. 48, no. 3, pp. 515-527, 2008.

[21] C.-Y. Huang, Y.-J. Li, and H.-F. Huo, "The dynamics of a stage-structured predator-prey system with impulsive effect and Holling mass defence," Applied Mathematical Modelling, vol. 36, no. 1, pp. 87-96, 2012.

[22] Y. Z. Wang and M. Zhao, "Dynamic analysis of an impulsively controlled predator-prey model with Holling type IV functional response," Discrete Dynamics in Nature and Society, vol. 2012, Article ID 141272, 18 pages, 2012.
[23] H.-F. Huo and W.-T. Li, "Dynamics of a nonautonomous semiratio-dependent predator-prey system with nonmonotonic functional responses," Discrete Dynamics in Nature and Society, vol. 2006, Article ID 70656, 19 pages, 2006.

[24] X. P. Li and W. S. Yang, "Permanence of a semi-ratio-dependent predator-prey system with nonmonotonic functional response and time delay," Abstract and Applied Analysis, vol. 2009, Article ID 960823, 6 pages, 2009.

[25] S. W. Zhang, L. Z. Dong, and L. S. Chen, “The study of predatorprey system with defensive ability of prey and impulsive perturbations on the predator," Chaos, Solitons \& Fractals, vol. 23, no. 2, pp. 631-643, 2005.

[26] Y. Z. Pei, C. G. Li, L. S. Chen, and C. Wang, "Complex dynamics of one-prey multi-predator system with defensive ability of prey and impulsive biological control on predators," Advances in Complex Systems, vol. 8, no. 4, pp. 483-495, 2005.

[27] S. Y. Li, Z. L. Xiong, and X. Wang, "The study of a predatorprey system with group defense and impulsive control strategy," Applied Mathematical Modelling, vol. 34, no. 9, pp. 2546-2561, 2010.

[28] D. D. Bainov and D. D. Simeonov, Impulsive Differential Equations: Periodic Solutions and Applications, Longman UK, 1993.

[29] V. Lakshmikantham, D. D. Bainnov, and P. S. Simeonov, Theory of Impulsive Differential Equations, vol. 6, World Scientific Publishing, Teaneck, NJ, USA, 1989.

[30] A. Lakmeche and O. Arino, "Bifurcation of non trivial periodic solutions of impulsive differential equations arising chemotherapeutic treatment," Dynamics of Continuous, Discrete and Impulsive Systems, vol. 7, no. 2, pp. 265-287, 2000. 


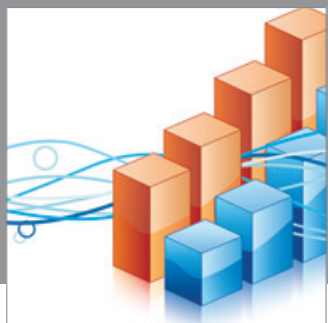

Advances in

Operations Research

mansans

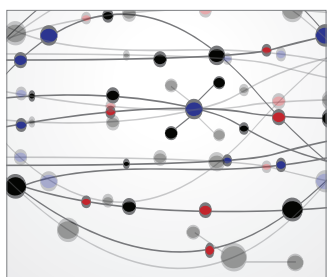

The Scientific World Journal
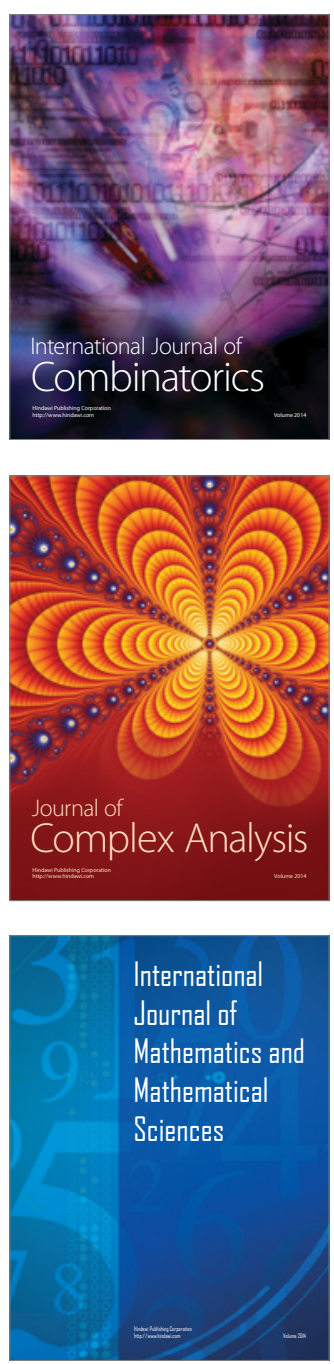
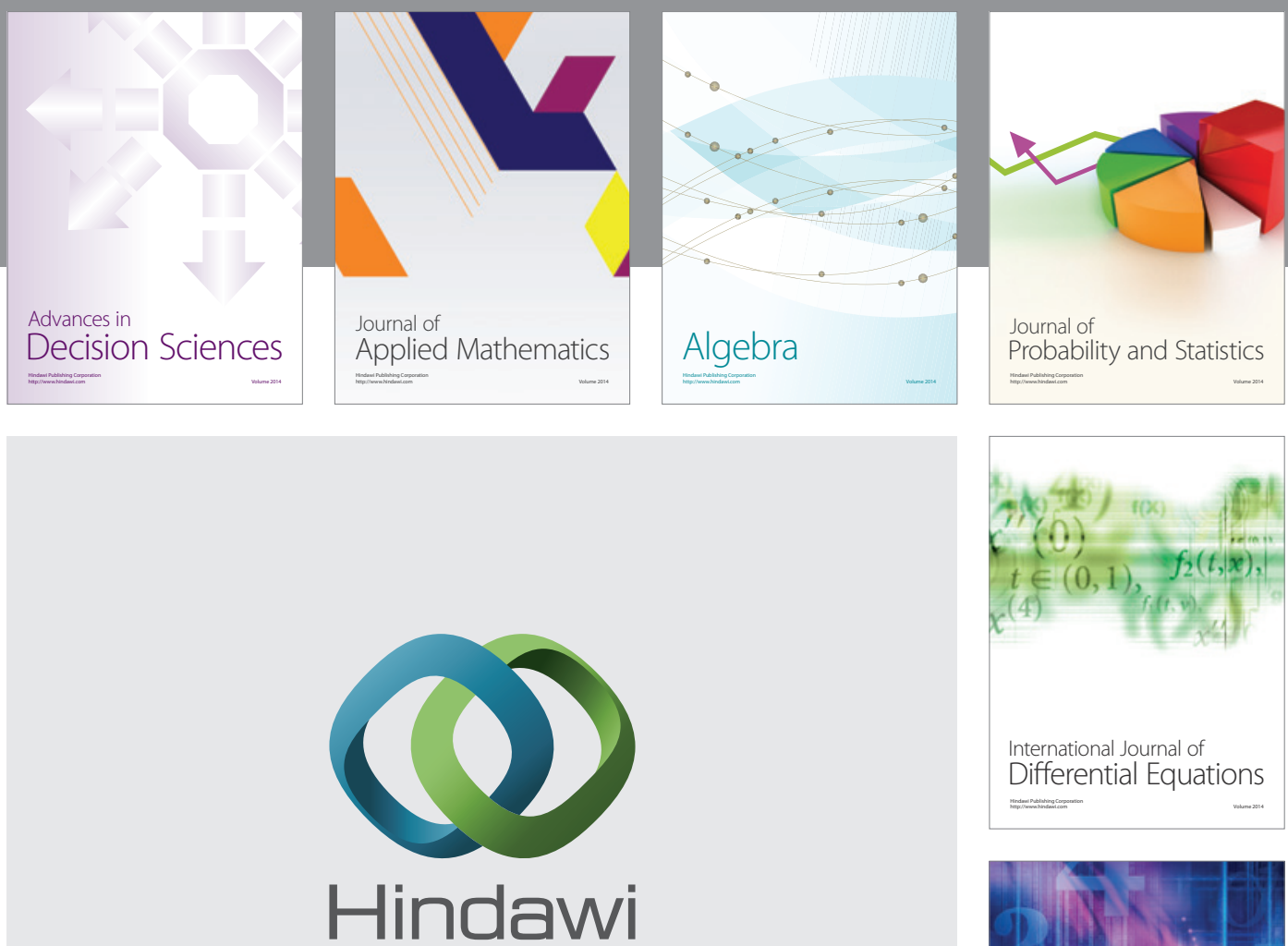

Submit your manuscripts at http://www.hindawi.com
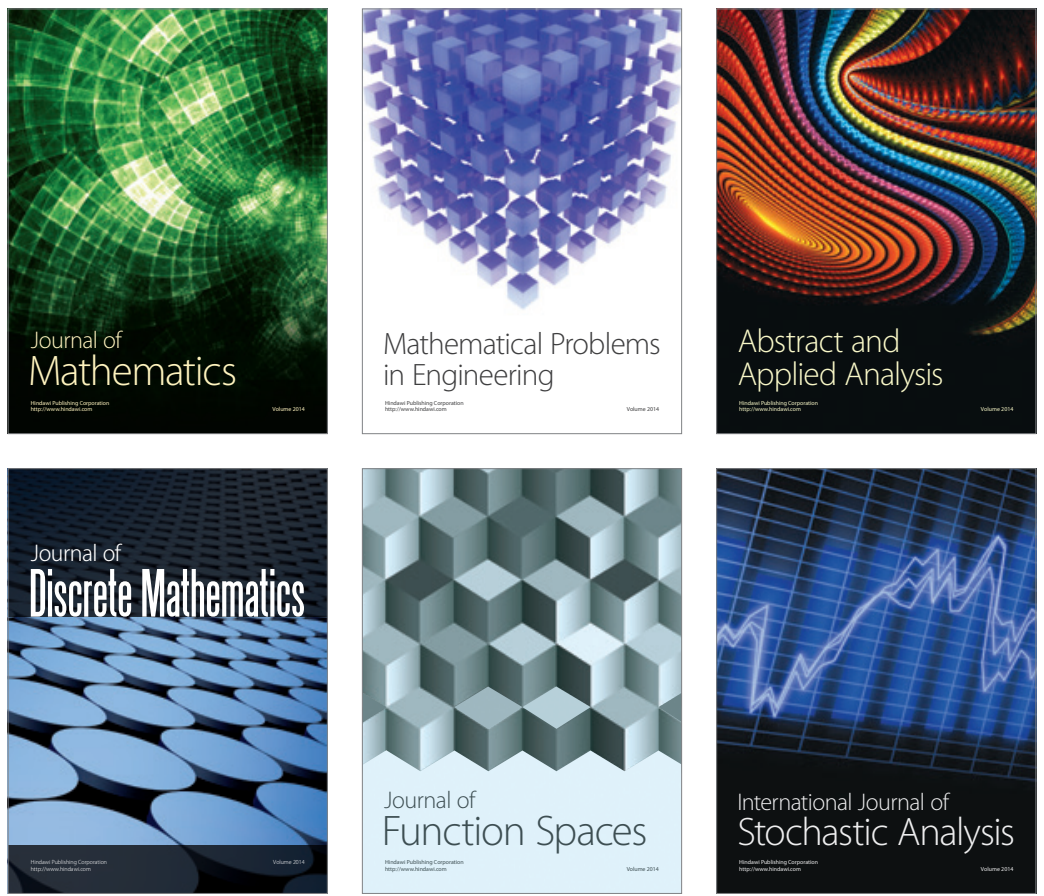

Journal of

Function Spaces

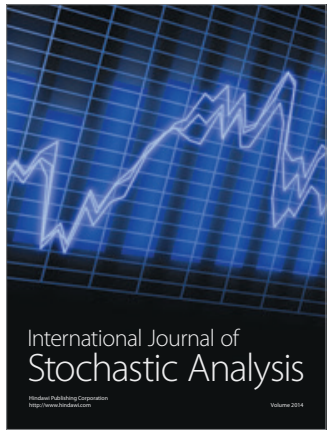

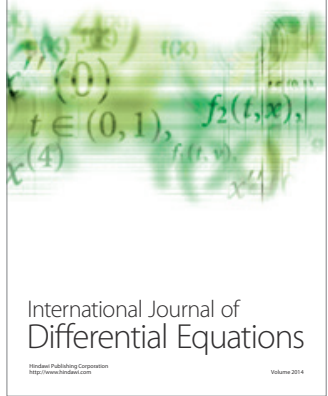
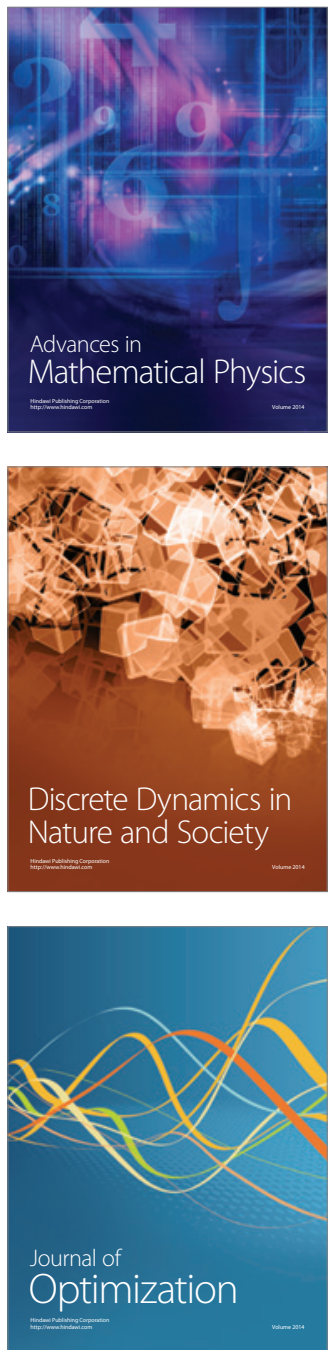